Prepared in cooperation with the Arkansas Natural Resources Commission

\title{
Water Use in Arkansas, 2005
}

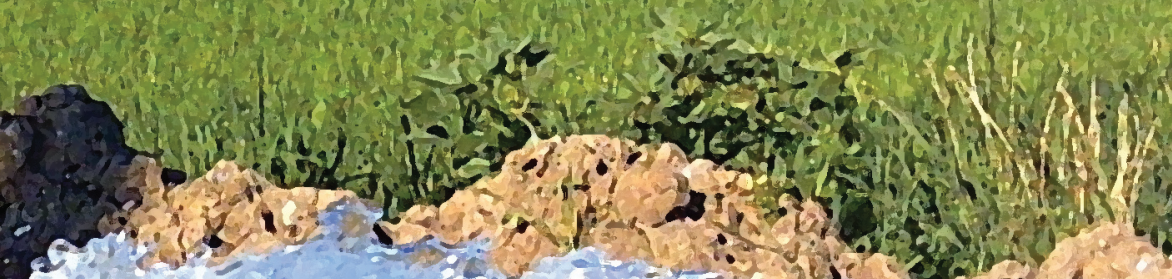

ateng

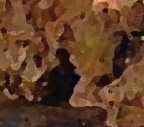

Scientific Investigations Report 2007-5241

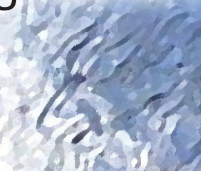

U.S. Department of the Interior

U.S. Geological Survey
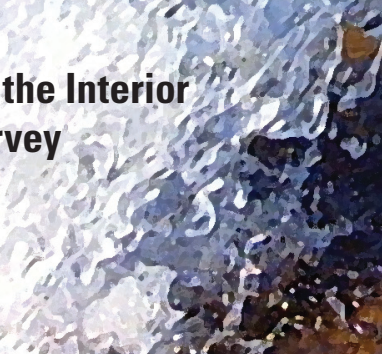

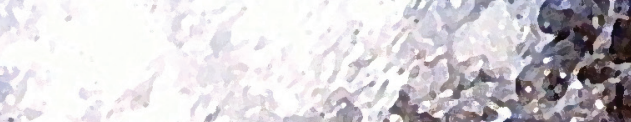

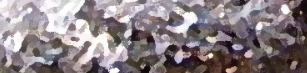

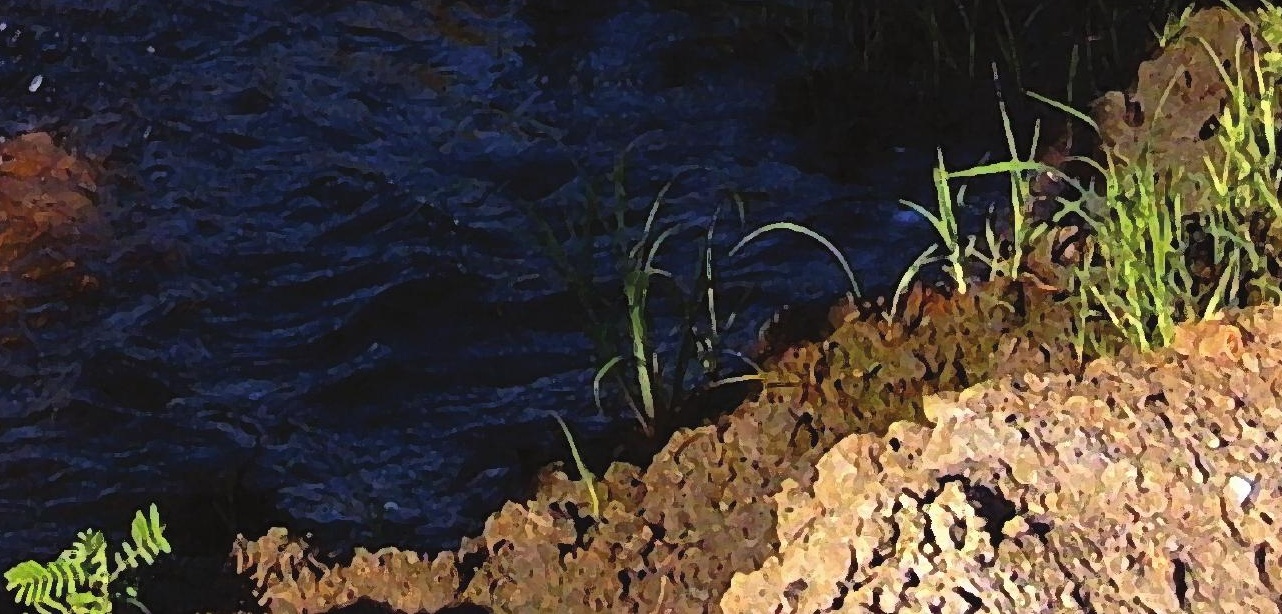


Cover photograph. Irrigation well in northeastern Arkansas (photograph by Ralf Montanus, U.S. Geological Survey, 2007). 


\section{Water Use in Arkansas, 2005}

By Terrance W. Holland

In cooperation with the Arkansas Natural Resources Commission

Scientific Investigations Report 2007-5241 


\section{U.S. Department of the Interior DIRK KEMPTHORNE, Secretary}

\section{U.S. Geological Survey \\ Mark D. Myers, Director}

\section{U.S. Geological Survey, Reston, Virginia: 2007}

For product and ordering information:

World Wide Web: http://www.usgs.gov/pubprod

Telephone: 1-888-ASK-USGS

For more information on the USGS--the Federal source for science about the Earth, its natural and living resources, natural hazards, and the environment:

World Wide Web: http://www.usgs.gov

Telephone: 1-888-ASK-USGS

Any use of trade, product, or firm names is for descriptive purposes only and does not imply endorsement by the U.S. Government.

Although this report is in the public domain, permission must be secured from the individual copyright owners to reproduce any copyrighted materials contained within this report.

Suggested citation:

Holland, T.W., 2007, Water use in Arkansas, 2005: U.S. Geological Survey Scientific Investigations Report 2007-5241, $32 \mathrm{p}$. 


\section{Contents}

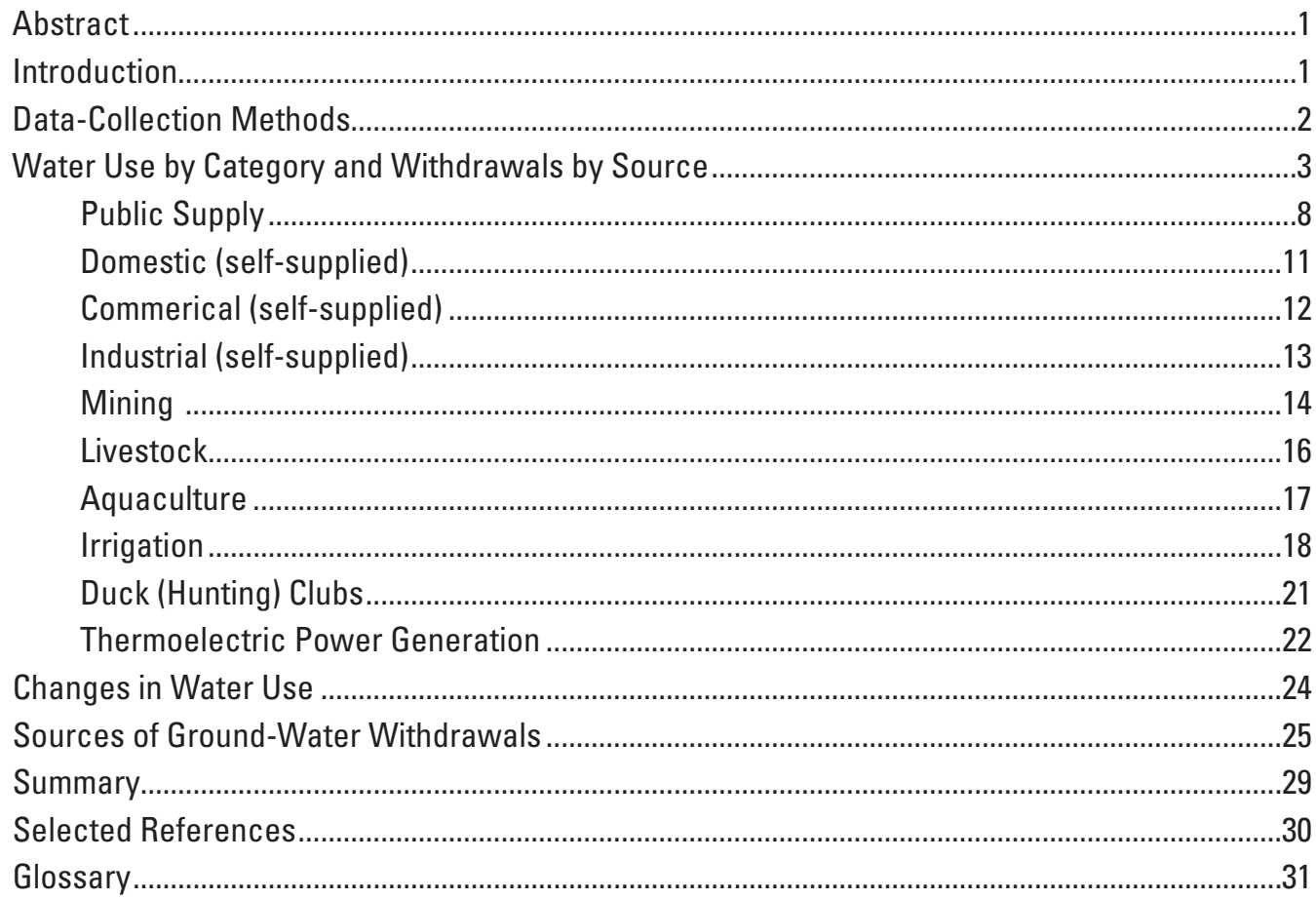

\section{Figures}

1-5. Maps showing:

1. Major surface-water sources ...................................................................................

2. Counties where selected water-use data are reported at Conservation District

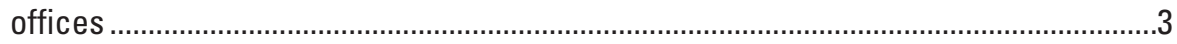

3. Total water use by county for Arkansas, 2005 ..........................................................

4. Ground-water use by county for Arkansas, 2005 ....................................................

5. Surface-water use by county for Arkansas, 2005 ....................................................

6-10. Graphs showing:

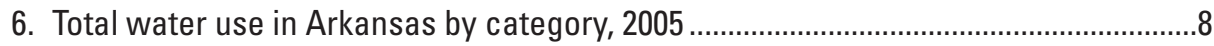

7. Ground-water use in Arkansas by category, 2005 ................................................

8. Surface-water use in Arkansas by category, 2005 .....................................................

9. Total ground- and surface-water use compared to irrigation water use in

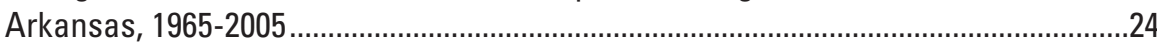

10. Ground- and surface-water use and population served for public-supply systems in Arkansas, 1965-2005

11. Maps showing location of selected major aquifers in Arkansas ........................................25 


\section{Tables}

1. Ground water, surface water, and total withdrawals by county for Arkansas, 2005..........4

2. Public supply and per capita water use in Arkansas, 2005 ..............................................

3. Domestic (self-supplied) water use in Arkansas, 2005.......................................................11

4. Commercial (self-supplied) water use in Arkansas, 2005 ...................................................12

5. Industrial (self-supplied) water use in Arkansas, 2005......................................................13

6. Mining water use in Arkansas, 2005...................................................................................... 15

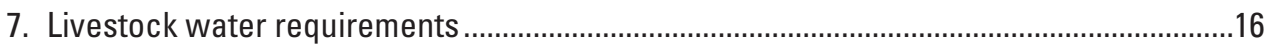

8. Livestock water use in Arkansas, 2005 ..................................................................................16

9. Aquaculture water use in Arkansas, 2005 ..............................................................................17

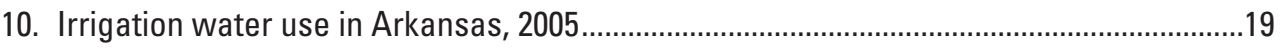

11. Duck (hunting) clubs water use in Arkansas, 2005 ...............................................................21

12. Thermoelectric-power generation water use in Arkansas, 2005 ......................................23

13. Changes in water use for selected categories in Arkansas, 1965-2005 ............................24

14. Ground-water use by major aquifers in Arkansas, 2005 ...................................................26

\section{Conversion Factors and Datums}

\begin{tabular}{lcl}
\hline \multicolumn{1}{c}{ Multiply } & By & \multicolumn{1}{c}{ To obtain } \\
\hline meter $(\mathrm{m})$ & 3.281 & foot $(\mathrm{ft})$ \\
cubic meter $\left(\mathrm{m}^{3}\right)$ & 35.31 & cubic foot $\left(\mathrm{ft}^{3}\right)$ \\
kilometer $(\mathrm{km})$ & 0.6214 & mile $(\mathrm{mi})$ \\
square kilometer $\left(\mathrm{km}^{2}\right)$ & 247.1 & square mile $\left(\mathrm{mi}^{2}\right)$ \\
cubic meter per second $\left(\mathrm{m}^{3} / \mathrm{s}\right)$ & 35.31 & cubic foot per second $\left(\mathrm{ft}^{3} / \mathrm{s}\right)$ \\
kilogram $(\mathrm{kg})$ & 2.205 & pound $(\mathrm{lb})$ \\
\hline
\end{tabular}

Temperature in degrees Celsius $\left({ }^{\circ} \mathrm{C}\right)$ may be converted to degrees Fahrenheit $\left({ }^{\circ} \mathrm{F}\right)$ as follows:

$$
{ }^{\circ} \mathrm{F}=\left(1.8 \mathrm{x}^{\circ} \mathrm{C}\right)+32
$$

Vertical coordinate information is referenced to the National Geodetic Vertical Datum of 1929 (NGVD of 1929). 


\title{
Water Use in Arkansas, 2005
}

\author{
By Terrance W. Holland
}

\section{Abstract}

The water-use program in Arkansas is a cooperative effort between the Arkansas Natural Resources Commission and the U.S. Geological Survey to inventory water use. During 2005, the amount of water withdrawn from ground- and surface-water sources in Arkansas was estimated to be 11,455 million gallons per day (Mgal/d). Of this amount, about 7,510 $\mathrm{Mgal} / \mathrm{d}$ (66 percent) was from ground-water and about 3,946 $\mathrm{Mgal} / \mathrm{d}$ (34 percent) was from surface-water sources.

Approximately 93 percent of the population (2.6 million people) in Arkansas was served by public supply systems during 2005. These systems withdrew approximately $404 \mathrm{Mgal} / \mathrm{d}$. Most of the water, 66 percent, was from surface-water sources. The statewide average for per-capita residential use from public supply systems was 157 gallons per day and increased about 35 percent between 1965 and 2005.

The largest use of water was for irrigation $(8,265$ $\mathrm{Mgal} / \mathrm{d}$ ), which accounted for 92 percent (6,942 Mgal/d) of the ground water withdrawn in Arkansas and 72 percent of the total withdrawals (both ground water and surface water). The next largest use category is thermoelectric generation $(1,997$ $\mathrm{Mgal} / \mathrm{d}$ ), followed by public supply (404 Mgal/d) and duck (hunting) clubs (269 Mgal/d). The withdrawal categories of domestic, commercial, industrial, mining, livestock, and aquaculture each withdrew less than $260 \mathrm{Mgal} / \mathrm{d}$.

\section{Introduction}

Water is one of Arkansas' most valuable natural resources. Much of the State's agriculture and industry is dependent upon having an adequate supply of quality water. The Arkansas Natural Resources Commission (ANRC) conducts an annual inventory of the ground- and surface-water withdrawals in Arkansas in cooperation with the U.S. Geological Survey (USGS). Data collected during this inventory are shared by State and Federal agencies to document the State's total water use and to facilitate planning the most effective use of Arkansas' water resources for the economic and social well being of the people of Arkansas and the Nation.

Every 5 years since 1950, the USGS has conducted an inventory of water use in the United States (MacKichan, 1951, 1957; MacKichan and Kammerer, 1961; Murray, 1968; Murray and Reeves, 1972, 1977; Solley and others, 1983, 1988, 1993, 1998; Hutson and others, 2004). In 1978, the USGS initiated the National Water-Use Information Program to establish a nationwide water-use database. In 1985, the ANRC and the USGS began a water-use program that provides for the collection, storage, and dissemination of accurate water-use information for Arkansas within a consistent national framework.

Ground- and surface-water sources are important in Arkansas. Major aquifers are present in alluvial deposits and in the Cockfield Formation, Cane River Formation, Sparta Sand, and Memphis Sand of Claiborne Group; Wilcox Group (undifferentiated); Nacatoch Sand; Tokio Formation; Trinity Group; and rocks of Paleozoic age (undifferentiated) (Petersen and others, 1985). Hereafter, these aquifers are referred to as the Mississippi River Valley alluvial aquifer, aquifers in other alluvial deposits, Cockfield aquifer, Cane River aquifer, Sparta-Memphis aquifer, Wilcox aquifer, Claiborne aquifer, Nacatoch aquifer, Tokio aquifer, Trinity aquifer, and Paleozoic aquifer. Major surface-water sources include the Arkansas, Saline, and Ouachita Rivers, and Beaver, Bull Shoals, Maumelle, and Winona Lakes (fig. 1).

This report describes water use in Arkansas based on the total withdrawals from ground- and surface-water resources by county for 2005. Ten categories of water use are described in this report-public supply, domestic (self-supplied), commercial (self-supplied), industrial (self-supplied), mining, livestock, aquaculture, irrigation, duck (hunting) clubs, and thermoelectric power generation. Water-use trends in Arkansas from 1965 to 2005 and sources of ground-water withdrawals also are described. 


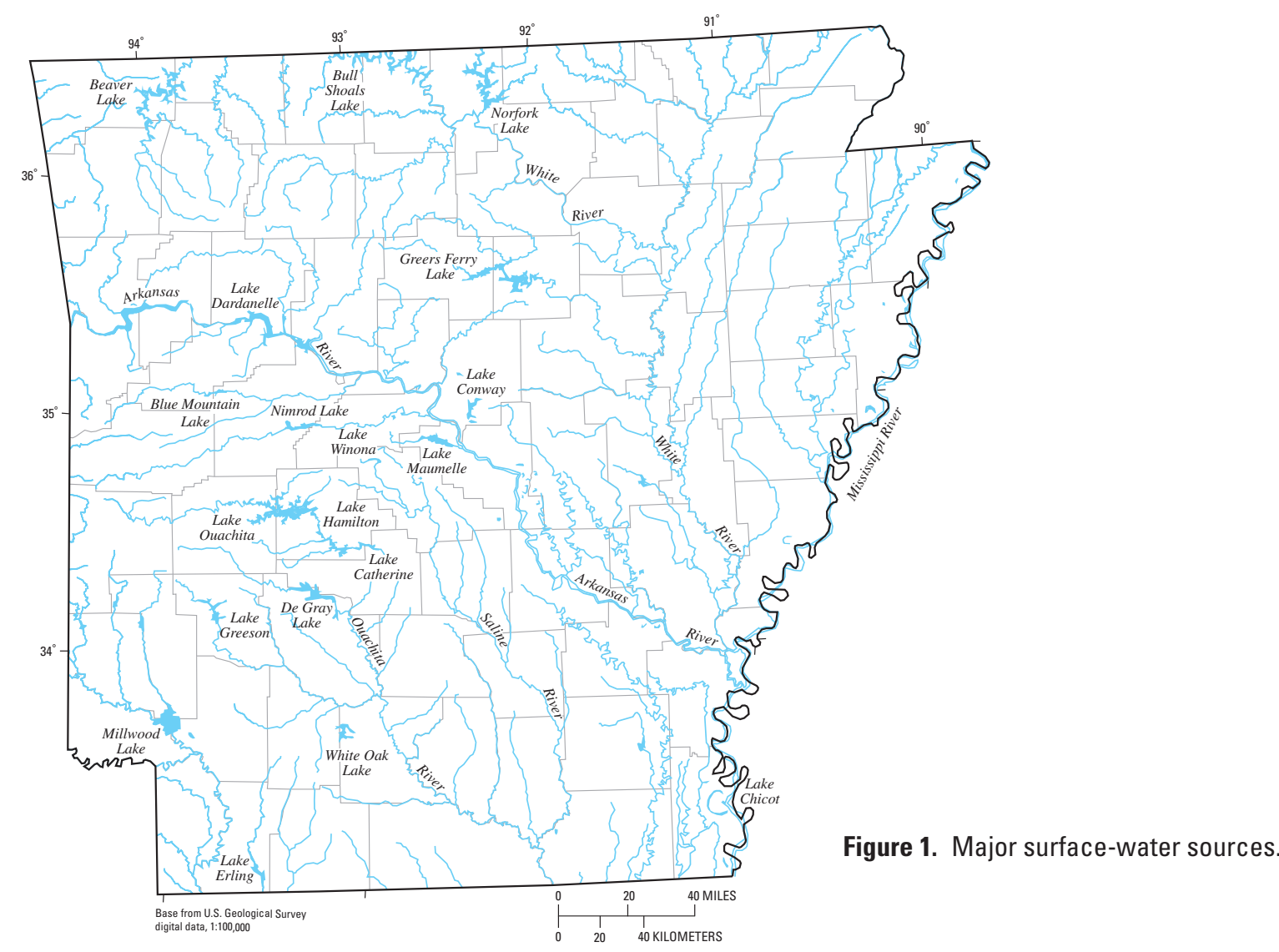

\section{Data-Collection Methods}

Site-specific water-use data for several categories are collected and compiled annually by the ANRC in cooperation with the USGS. Water users that withdraw 1 acre-foot or more of surface water per year or wells with the capability of pumping 50,000 gallons per day (gal/d) or more of ground water are required by Arkansas law to report their withdrawals to the appropriate reporting agency. Data for the irrigation, livestock, aquaculture, and duck (hunting) clubs categories are reported through the Conservation District offices in selected counties (fig. 2). Water-use data for each of the other categories are reported directly to the ANRC. Site-specific water-use data for irrigation, livestock, duck (hunting) clubs, public supply, commercial, industrial, mining, and power generation are stored in the Arkansas Water-Use Data Base (WUDBS) maintained by USGS (http://ar.water.usgs.gov/wateruse.html). Information about amounts of water withdrawn, sources of water, how the water was used, and how much water was returned is available to water-resources managers and policy makers through WUDBS.

Because of incomplete reporting, in some cases it is necessary to supplement these data with data from other sources. These sources include public agencies, industries, public utilities, other organizations, and individuals. Principal contributors include the Arkansas Agricultural Experiment Station, Arkansas Department of Environmental Quality, Arkansas
Department of Health, Arkansas Department of Parks and Tourism, Arkansas Electric Cooperative, Arkansas Geological Survey, Arkansas Industrial Development Commission, Entergy Arkansas, Arkansas State Highway and Transportation Department, the Cooperative Extension Service, National Agricultural Statistics Service, Natural Resources Conservation Service, Southwest Power Administration, U.S. Army Corps of Engineers, U.S. Department of Energy, and the U.S. Fish and Wildlife Service.

Estimates are made by the USGS based on population (U.S. Bureau of Commerce, 2005) and average consumptiveuse rates (Holland, 1987) for water-use categories for which data are not available or are incomplete. After sufficient data are collected from all available sources, these data are aggregated by county for each water-use category and by aquifer for each county. 


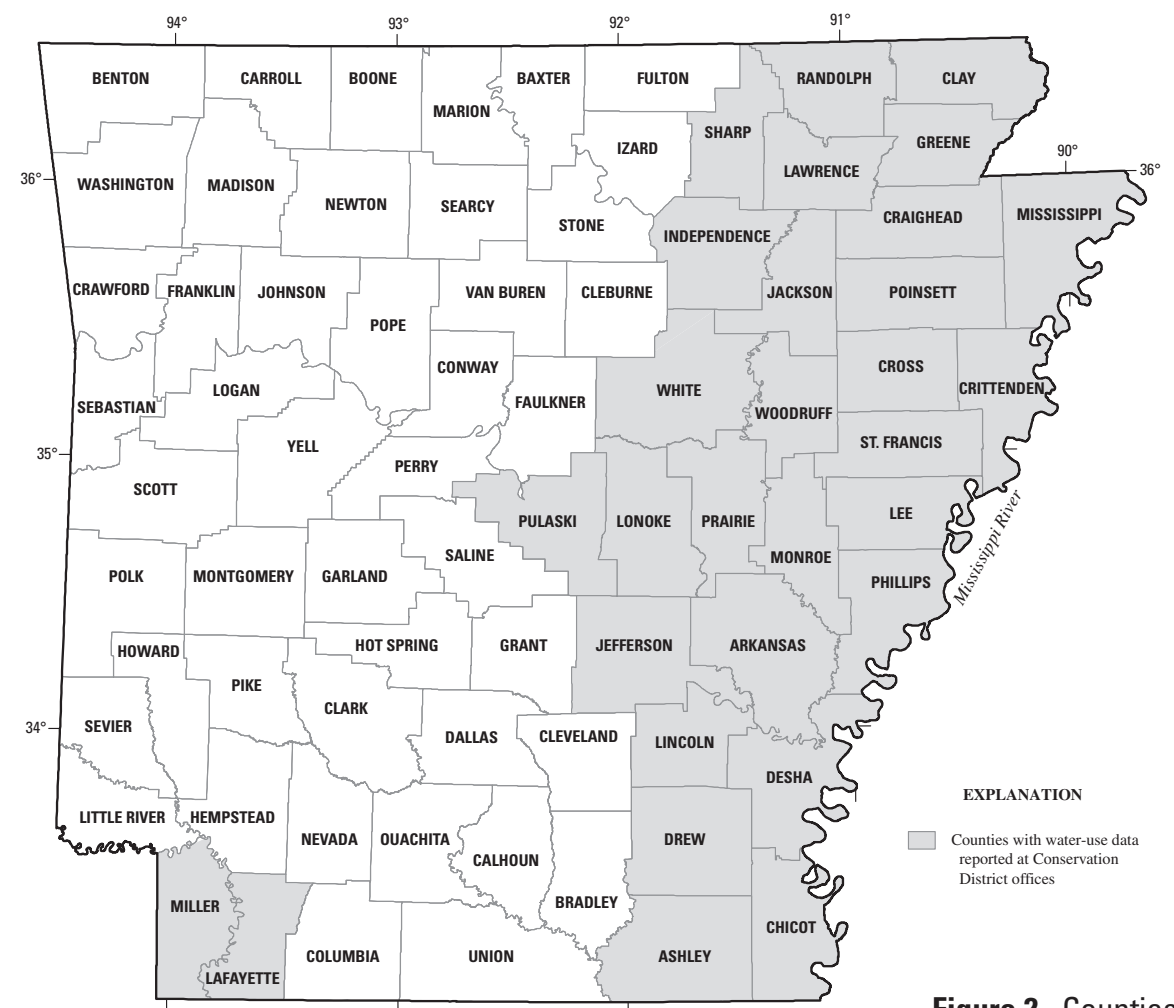

Figure 2. Counties where selected water-use data are reported at Conservation District offices.

\section{Water Use by Category and Withdrawals by Source}

During 2005, total withdrawals from ground-water and surface-water sources in Arkansas were 11,455 Mgal/d (table 1 , most water-use values listed in discussions in rest of report are rounded to whole numbers). This is an increase of about 31 percent from the total of $8,767 \mathrm{Mgal} / \mathrm{d}$ withdrawn in 1995 (Holland, 1999) and about a 4.5 percent increase from that used (10,963 Mgal/d) in 2000 (Holland, 2004). Of the total withdrawn in 2005 , about 66 percent $(7,510 \mathrm{Mgal} / \mathrm{d})$ was from ground water, and the remaining 34 percent $(3,946 \mathrm{Mgal} / \mathrm{d})$ was from surface water. The largest total withdrawals generally occurred in eastern Arkansas and in counties along major rivers (fig. 3). The five counties with the largest total water use are Pope, Arkansas, Poinsett, Cross, and Lonoke, in order of decreasing use (table 1). The largest ground-water users were in eastern Arkansas (fig. 4). The highest ground-water use (682 Mgal/d) occurred in Poinsett County primarily for irrigation (table 1). The highest surface-water use occurred from the Arkansas River in Pope County primarily for thermoelectric power generation (1,163 Mgal/d) (table 1, fig. 5).
The distribution of total (both ground and surface) water use by category (fig. 6) illustrates the dominance of water use for irrigation in Arkansas. Irrigation also is the dominant category of ground-water use in Arkansas (fig. 7). Duck (hunting) club water use (269 Mgal/d) is the fourth largest category in Arkansas. Thermoelectric power generation, followed by irrigation, is the dominant category of surface-water use in Arkansas (fig. 8). 
Table 1. Ground water, surface water, and total withdrawals by county for Arkansas, 2005.

[Mgal/d, million gallons per day]

\begin{tabular}{|c|c|c|c|c|c|c|c|}
\hline County & $\begin{array}{c}\text { Ground water } \\
\text { (Mgal/d) }\end{array}$ & $\begin{array}{c}\text { Surface water } \\
\text { (Mgal/d) }\end{array}$ & $\begin{array}{c}\text { Total } \\
\text { (Mgal/d) }\end{array}$ & County & $\begin{array}{c}\text { Ground water } \\
\text { (Mgal/d) }\end{array}$ & $\begin{array}{c}\text { Surface water } \\
\text { (Mgal/d) }\end{array}$ & $\begin{array}{c}\text { Total } \\
\text { (Mgal/d) }\end{array}$ \\
\hline Arkansas & 509.48 & 457.64 & 967.12 & Lincoln & 182.87 & 23.53 & 206.40 \\
\hline Ashley & 156.30 & 75.97 & 232.27 & Little River & 4.43 & 10.04 & 14.47 \\
\hline Baxter & 1.95 & 3.88 & 5.83 & Logan & 1.65 & 4.25 & 5.90 \\
\hline Benton & 2.87 & 438.18 & 441.05 & Lonoke & 423.84 & 110.41 & 534.25 \\
\hline Boone & 1.91 & 1.19 & 3.10 & Madison & 1.15 & 0.98 & 2.13 \\
\hline Bradley & 1.78 & 0.08 & 1.86 & Marion & 0.83 & 1.21 & 2.04 \\
\hline Calhoun & 0.53 & 0.04 & 0.57 & Miller & 16.28 & 110.30 & 126.58 \\
\hline Carroll & 2.87 & 8.72 & 11.59 & Mississippi & 277.76 & 2.57 & 280.33 \\
\hline Chicot & 249.53 & 70.39 & 319.92 & Monroe & 290.04 & 26.41 & 316.45 \\
\hline Clark & 0.51 & 6.07 & 6.58 & Montgomery & 0.70 & 0.82 & 1.52 \\
\hline Clay & 471.05 & 10.20 & 481.25 & Nevada & 0.73 & 1.36 & 2.09 \\
\hline Cleburne & 0.41 & 8.35 & 8.76 & Newton & 1.17 & 0.23 & 1.40 \\
\hline Cleveland & 0.73 & 0.20 & 0.93 & Ouachita & 1.08 & 58.46 & 59.54 \\
\hline Columbia & 3.64 & 2.23 & 5.87 & Perry & 0.46 & 14.33 & 14.79 \\
\hline Conway & 2.51 & 16.90 & 19.41 & Phillips & 208.51 & 1.78 & 210.29 \\
\hline Craighead & 365.03 & 44.68 & 409.71 & Pike & 0.48 & 1.21 & 1.69 \\
\hline Crawford & 0.94 & 7.61 & 8.55 & Poinsett & 682.31 & 90.48 & 772.79 \\
\hline Crittenden & 159.51 & 0.97 & 160.48 & Polk & 1.17 & 2.39 & 3.56 \\
\hline Cross & 599.29 & 45.01 & 644.30 & Pope & 2.09 & $1,162.81$ & $1,164.90$ \\
\hline Dallas & 1.47 & 0.03 & 1.50 & Prairie & 253.58 & 102.18 & 355.76 \\
\hline Desha & 304.34 & 109.27 & 413.61 & Pulaski & 26.39 & 57.88 & 84.27 \\
\hline Drew & 77.40 & 13.41 & 90.81 & Randolph & 102.51 & 38.88 & 141.39 \\
\hline Faulkner & 2.40 & 14.81 & 17.21 & Saline & 2.29 & 6.98 & 9.27 \\
\hline Franklin & 0.40 & 3.52 & 3.92 & Scott & 2.35 & 0.45 & 2.80 \\
\hline Fulton & 1.96 & 0.48 & 2.44 & Searcy & 0.29 & 0.70 & 0.99 \\
\hline Garland & 0.32 & 19.56 & 19.88 & Sebastian & 0.94 & 38.46 & 39.40 \\
\hline Grant & 2.60 & 0.10 & 2.70 & Sevier & 1.74 & 0.82 & 2.56 \\
\hline Greene & 223.63 & 3.90 & 227.53 & Sharp & 4.31 & 0.98 & 5.29 \\
\hline Hempstead & 4.02 & 8.34 & 12.36 & St. Francis & 295.73 & 18.57 & 314.30 \\
\hline Hot Spring & 0.27 & 322.90 & 323.17 & Stone & 0.60 & 1.84 & 2.44 \\
\hline Howard & 0.50 & 5.14 & 5.64 & Union & 15.55 & 10.65 & 26.20 \\
\hline Independence & 41.84 & 62.74 & 104.58 & Van Buren & 0.41 & 2.60 & 3.01 \\
\hline Izard & 1.79 & 3.22 & 5.01 & Washington & 1.35 & 2.50 & 3.85 \\
\hline Jackson & 383.12 & 22.58 & 405.70 & White & 48.59 & 42.93 & 91.52 \\
\hline Jefferson & 279.71 & 70.27 & 349.98 & Woodruff & 267.00 & 84.47 & 351.47 \\
\hline Johnson & 0.32 & 5.02 & 5.34 & Yell & 2.37 & 7.42 & 9.79 \\
\hline Lafayette & 39.40 & 1.81 & 41.21 & & & & \\
\hline Lawrence & 223.27 & 32.83 & 256.10 & & & & \\
\hline Lee & 266.79 & 5.41 & 272.20 & Total & $7,509.94$ & $3,945.53$ & $11,455.47$ \\
\hline
\end{tabular}




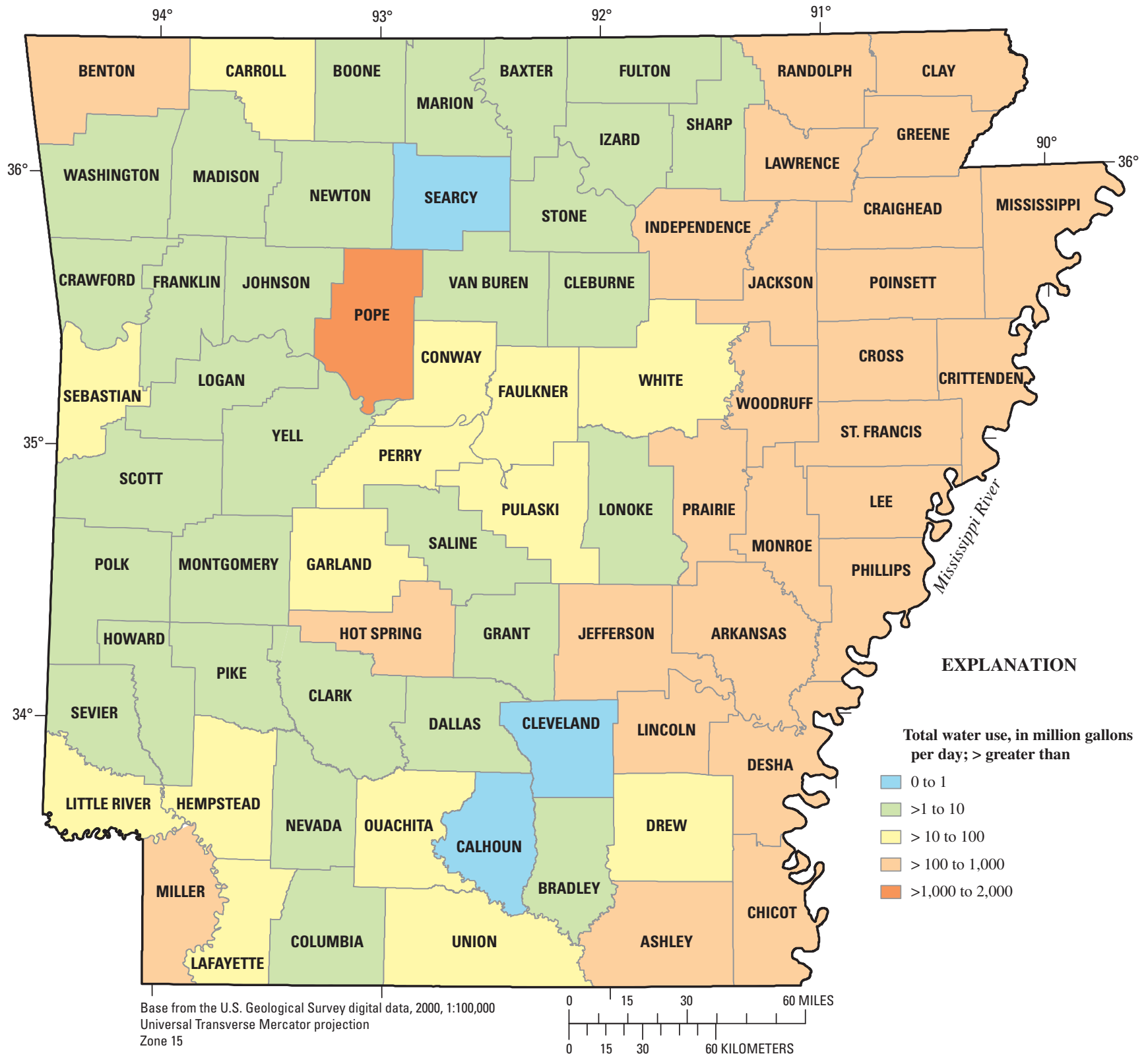

Figure 3. Total water use by county for Arkansas, 2005. 


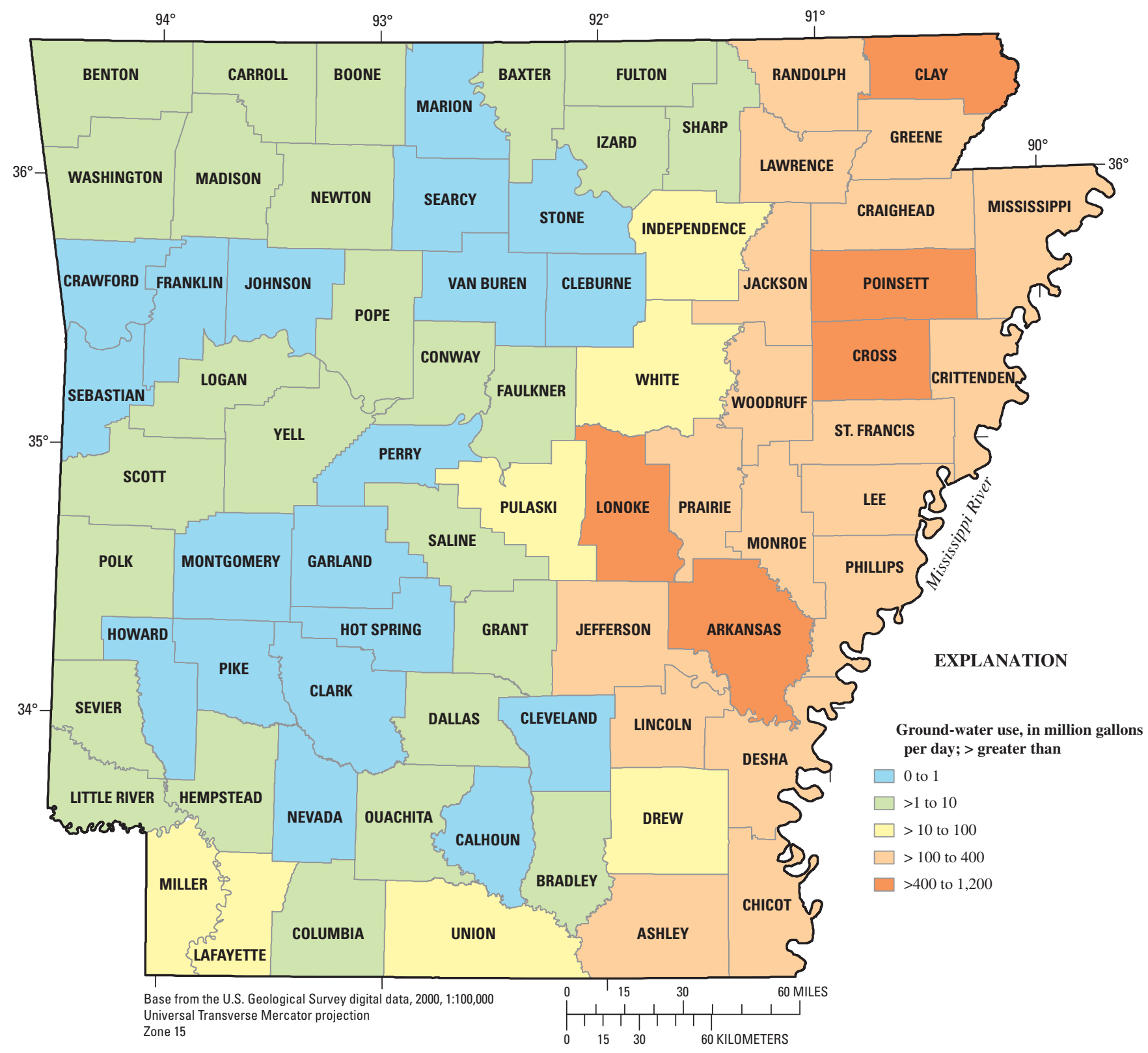

Figure 4. Ground-water use by county for Arkansas, 2005. 


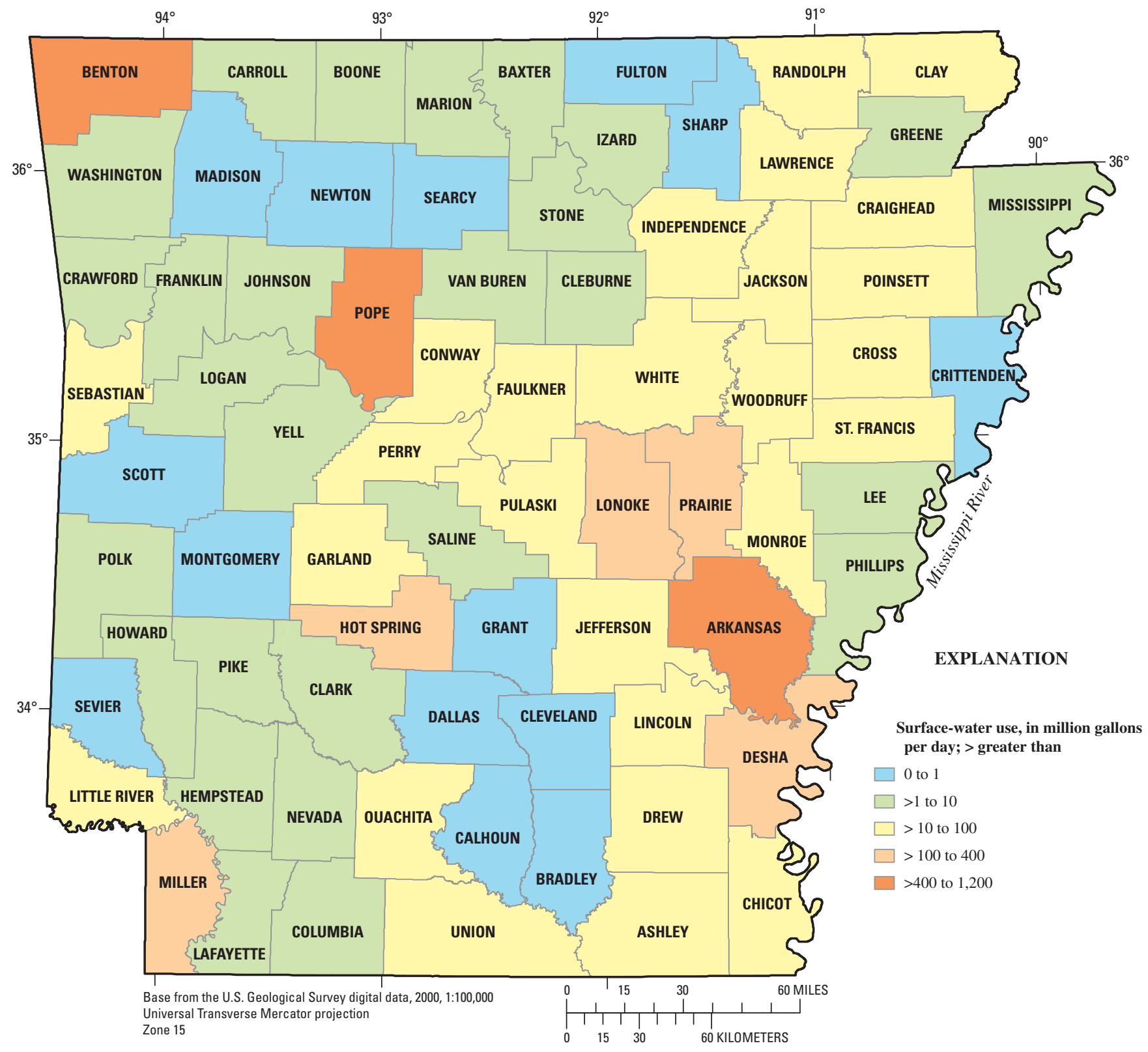

Figure 5. Surface-water use by county for Arkansas, 2005. 

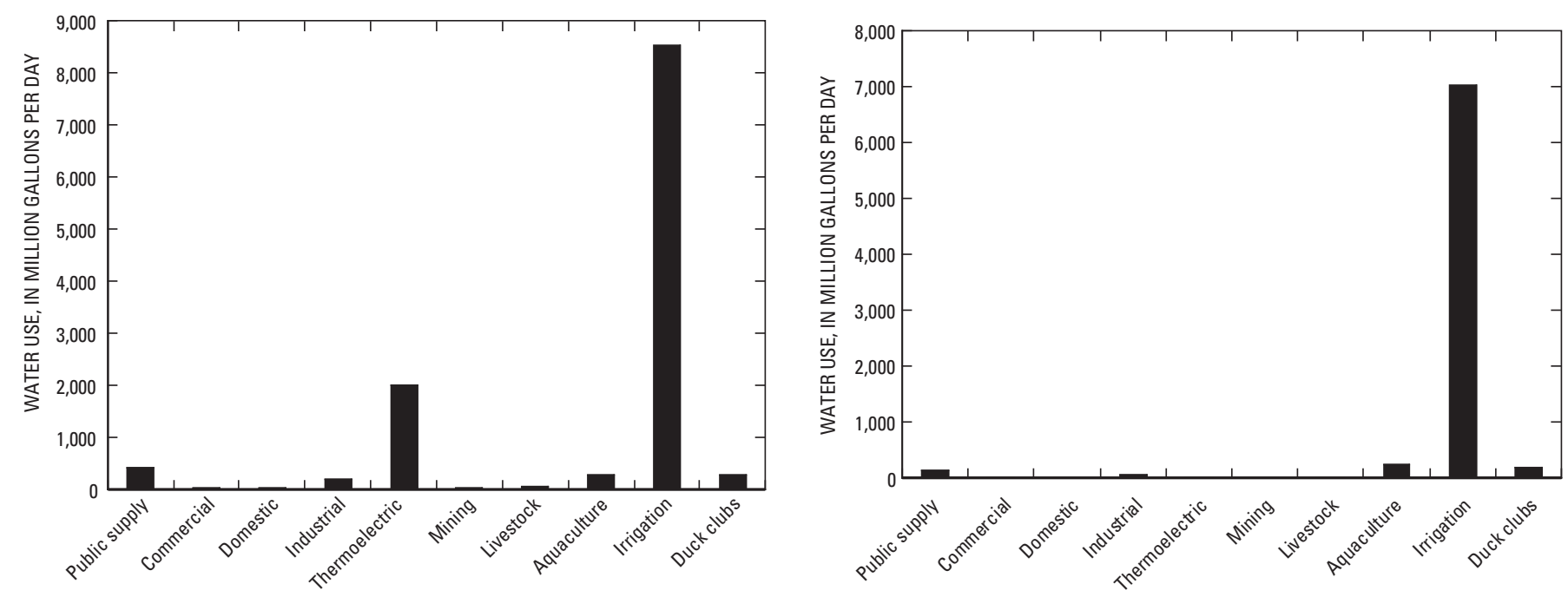

Figure 6. Total water use in Arkansas by category, 2005.

Figure 7. Ground-water use in Arkansas by category, 2005.

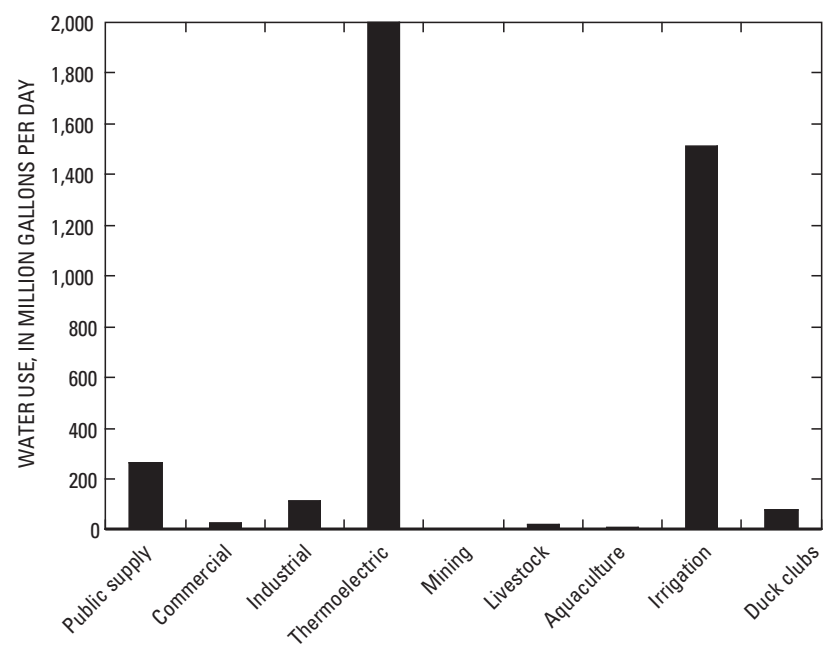

Figure 8. Surface-water use in Arkansas by category, 2005.

\section{Public Supply}

Public supply refers to water withdrawn by public and private water suppliers and delivered to multiple users for domestic, commercial, industrial, and thermoelectric power generation uses. Public supply includes public and private systems that furnish water to at least 25 people or have a minimum of 15 service connections.

Data on public-supply withdrawals are obtained from ANRC water-use registration reports. ANRC requires all public and private suppliers in Arkansas to report their annual and monthly withdrawals and deliveries. Site-specific data for about 800 public-water suppliers are stored in the WUDBS maintained by USGS. Data reporting deficiencies are supplemented with information from monthly operation reports reported to the Arkansas Department of Health (ADH).
Population served, source of water, water sold to facilities, and water purchased from other public supply facilities are available on the ADH web site (Arkansas Department of Health, 2007).

Public-supply systems served about 2.6 million people or about 93 percent of Arkansas' population in 2005. Public-supply withdrawals in 2005 in Arkansas were $404 \mathrm{Mgal} / \mathrm{d}$ (table 2; fig. 6); about 66 percent were from surface-water sources. Public-supply withdrawals account for about 4 percent of the total withdrawals of water in the State for all purposes. Ground-water withdrawals for public supply were about 2 percent of the State's total ground-water withdrawals for 2005. Public-supply surface-water withdrawals were about 7 percent of the State's total surface-water withdrawals.

Benton County had the largest total withdrawals for public supply at $59 \mathrm{Mgal} / \mathrm{d}$ during 2005. Jefferson County had the 
Table 2. Public supply and per capita water use in Arkansas, 2005.

[Mgal/d, million gallons per day; gal/d, gallons per day]

\begin{tabular}{|c|c|c|c|c|c|}
\hline \multirow[b]{2}{*}{ County } & \multirow{2}{*}{$\begin{array}{c}\text { Total population } \\
\text { served } \\
\text { (thousands) }\end{array}$} & \multicolumn{3}{|c|}{$\begin{array}{c}\text { Water withdrawals } \\
\text { (Mgal/d) }\end{array}$} & \multirow{2}{*}{$\begin{array}{c}\text { Per capita } \\
\text { water use } \\
\text { (gal/d) }\end{array}$} \\
\hline & & $\begin{array}{l}\text { Ground } \\
\text { water }\end{array}$ & $\begin{array}{c}\text { Surface } \\
\text { water }\end{array}$ & Total & \\
\hline Arkansas & 18.07 & 6.02 & 0.00 & 6.02 & 333 \\
\hline Ashley & 21.12 & 2.12 & 0.00 & 2.12 & 100 \\
\hline Baxter & 32.90 & 0.53 & 3.67 & 4.20 & 128 \\
\hline Benton & 181.20 & 1.10 & 57.99 & 59.09 & 326 \\
\hline Boone & 30.34 & 0.88 & 0.33 & 1.21 & 40 \\
\hline Bradley & 11.64 & 1.22 & 0.00 & 1.22 & 105 \\
\hline Calhoun & 4.50 & 0.40 & 0.00 & 0.40 & 89 \\
\hline Carroll & 23.59 & 0.90 & 7.71 & 8.61 & 365 \\
\hline Chicot & 11.03 & 1.82 & 0.00 & 1.82 & 165 \\
\hline Clark & 22.13 & 0.07 & 2.82 & 2.89 & 131 \\
\hline Clay & 16.58 & 1.42 & 0.00 & 1.42 & 86 \\
\hline Cleburne & 24.39 & 0.08 & 7.82 & 7.90 & 324 \\
\hline Cleveland & 8.10 & 0.54 & 0.00 & 0.54 & 67 \\
\hline Columbia & 24.00 & 1.38 & 1.96 & 3.34 & 139 \\
\hline Conway & 18.74 & 0.00 & 4.00 & 4.00 & 213 \\
\hline Craighead & 81.61 & 13.52 & 0.00 & 13.52 & 166 \\
\hline Crawford & 50.36 & 0.00 & 5.82 & 5.82 & 116 \\
\hline Crittenden & 49.88 & 8.47 & 0.00 & 8.47 & 170 \\
\hline Cross & 19.24 & 2.44 & 0.00 & 2.44 & 127 \\
\hline Dallas & 7.26 & 1.34 & 0.00 & 1.34 & 185 \\
\hline Desha & 14.36 & 2.18 & 0.00 & 2.18 & 152 \\
\hline Drew & 17.94 & 2.78 & 0.00 & 2.78 & 155 \\
\hline Faulkner & 91.85 & 0.52 & 8.03 & 8.55 & 93 \\
\hline Franklin & 18.22 & 0.00 & 2.89 & 2.89 & 159 \\
\hline Fulton & 10.95 & 1.28 & 0.00 & 1.28 & 117 \\
\hline Garland & 92.77 & 0.13 & 16.55 & 16.68 & 180 \\
\hline Grant & 15.96 & 2.03 & 0.00 & 2.03 & 127 \\
\hline Greene & 38.87 & 4.23 & 0.00 & 4.23 & 109 \\
\hline Hempstead & 14.65 & 2.65 & 0.43 & 3.08 & 210 \\
\hline Hot Spring & 29.50 & 0.00 & 3.64 & 3.64 & 123 \\
\hline Howard & 14.01 & 0.01 & 3.31 & 3.32 & 237 \\
\hline Independence & 32.83 & 0.87 & 5.52 & 6.39 & 195 \\
\hline Izard & 11.85 & 1.01 & 0.00 & 1.01 & 85 \\
\hline Jackson & 16.01 & 1.52 & 0.00 & 1.52 & 95 \\
\hline Jefferson & 80.38 & 14.25 & 0.00 & 14.25 & 177 \\
\hline Johnson & 24.04 & 0.00 & 4.58 & 4.58 & 191 \\
\hline Lafayette & 5.85 & 0.92 & 0.00 & 0.92 & 157 \\
\hline Lawrence & 17.15 & 1.61 & 0.00 & 1.61 & 94 \\
\hline Lee & 11.42 & 0.99 & 0.00 & 0.99 & 87 \\
\hline
\end{tabular}


Table 2. Public supply and per capita water use in Arkansas, 2005.-Continued

[Mgal/d, million gallons per day; gal/d, gallons per day]

\begin{tabular}{|c|c|c|c|c|c|}
\hline \multirow[b]{2}{*}{ County } & \multirow{2}{*}{$\begin{array}{c}\text { Total population } \\
\text { served } \\
\text { (thousands) }\end{array}$} & \multicolumn{3}{|c|}{$\begin{array}{l}\text { Water withdrawals } \\
\text { (Mgal/d) }\end{array}$} & \multirow{2}{*}{$\begin{array}{c}\text { Per capita } \\
\text { water use } \\
\text { (gal/d) }\end{array}$} \\
\hline & & $\begin{array}{l}\text { Ground } \\
\text { water }\end{array}$ & $\begin{array}{c}\text { Surface } \\
\text { water }\end{array}$ & Total & \\
\hline Lincoln & 14.26 & 1.04 & 0.00 & 1.04 & 73 \\
\hline Little River & 12.11 & 0.61 & 0.95 & 1.56 & 129 \\
\hline Logan & 13.24 & 0.00 & 3.36 & 3.36 & 254 \\
\hline Lonoke & 54.51 & 6.49 & 0.00 & 6.49 & 119 \\
\hline Madison & 8.27 & 0.00 & 0.00 & 0.00 & 0 \\
\hline Marion & 9.84 & 0.03 & 0.91 & 0.94 & 96 \\
\hline Miller & 35.02 & 0.25 & 0.00 & 0.25 & 7 \\
\hline Mississippi & 47.91 & 4.05 & 0.00 & 4.05 & 85 \\
\hline Monroe & 9.30 & 1.49 & 0.00 & 1.49 & 160 \\
\hline Montgomery & 3.95 & 0.03 & 0.48 & 0.51 & 129 \\
\hline Nevada & 6.79 & 0.15 & 0.77 & 0.92 & 135 \\
\hline Newton & 7.82 & 0.97 & 0.00 & 0.97 & 124 \\
\hline Ouachita & 26.48 & 0.97 & 2.81 & 3.78 & 143 \\
\hline Perry & 6.90 & 0.01 & 0.76 & 0.77 & 112 \\
\hline Phillips & 24.11 & 3.70 & 0.00 & 3.70 & 153 \\
\hline Pike & 8.42 & 0.05 & 0.85 & 0.90 & 107 \\
\hline Poinsett & 23.54 & 5.12 & 0.00 & 5.12 & 218 \\
\hline Polk & 10.87 & 0.00 & 1.74 & 1.74 & 160 \\
\hline Pope & 53.58 & 0.00 & 9.58 & 9.58 & 179 \\
\hline Prairie & 7.11 & 0.79 & 0.00 & 0.79 & 111 \\
\hline Pulaski & 366.46 & 4.56 & 44.67 & 49.23 & 134 \\
\hline Randolph & 13.01 & 0.33 & 1.15 & 1.48 & 114 \\
\hline Saline & 84.77 & 1.63 & 5.93 & 7.56 & 89 \\
\hline Scott & 7.14 & 1.69 & 0.00 & 1.69 & 237 \\
\hline Searcy & 7.14 & 0.00 & 0.38 & 0.38 & 53 \\
\hline Sebastian & 113.04 & 0.20 & 38.04 & 38.24 & 338 \\
\hline Sevier & 13.46 & 1.15 & 0.00 & 1.15 & 85 \\
\hline Sharp & 14.40 & 3.80 & 0.00 & 3.80 & 264 \\
\hline St. Francis & 25.90 & 5.18 & 0.00 & 5.18 & 200 \\
\hline Stone & 11.72 & 0.02 & 1.47 & 1.49 & 127 \\
\hline Union & 36.77 & 8.44 & 0.00 & 8.44 & 230 \\
\hline Van Buren & 16.03 & 0.00 & 2.36 & 2.36 & 147 \\
\hline Washington & 178.36 & 0.00 & 0.58 & 0.58 & 3 \\
\hline White & 63.13 & 0.75 & 8.27 & 9.02 & 143 \\
\hline Woodruff & 7.16 & 0.96 & 0.00 & 0.96 & 134 \\
\hline Yell & 21.39 & 2.00 & 4.28 & 6.28 & 294 \\
\hline Total & 2.579 .20 & 137.69 & 266.41 & 404.10 & ${ }^{1} 157$ \\
\hline
\end{tabular}

${ }^{1}$ Average per capita use for the State (in gallons per day). 
largest ground-water withdrawals for public supply with 14 $\mathrm{Mgal} / \mathrm{d}$, Benton County had the largest surface-water withdrawals for public supply (from Beaver Lake) with $58 \mathrm{Mgal} / \mathrm{d}$ during 2005. The statewide average for per capita residential use from public supply systems was about 157 gallons per day (gal/d). Carroll County had the highest per capita use at 365 $\mathrm{gal} / \mathrm{d}$ (table 2).

\section{Domestic (self-supplied)}

Domestic water use includes water for household purposes, such as drinking, food preparation, bathing, washing clothes and dishes, flushing toilets, and watering lawns and gardens. Most water used for domestic purposes in Arkansas is provided by public supply. However, about 199,000 people in Arkansas (or about 1 percent of the State's population) (U.S.

Table 3. Domestic (self-supplied) water use in Arkansas, 2005.

\begin{tabular}{|c|c|c|c|}
\hline County & $\begin{array}{c}\text { Population } \\
\text { (in thousands) }\end{array}$ & $\begin{array}{c}\text { Per capita } \\
\text { use } \\
\text { (gal/d) }\end{array}$ & $\begin{array}{c}\text { Domestic } \\
\text { use } \\
\text { (Mgal/d) }\end{array}$ \\
\hline Arkansas & 2.00 & 90 & 0.18 \\
\hline Ashley & 2.06 & 88 & 0.18 \\
\hline Baxter & 7.43 & 89 & 0.66 \\
\hline Benton & 5.74 & 89 & 0.51 \\
\hline Boone & 5.46 & 90 & 0.49 \\
\hline Bradley & 0.56 & 90 & 0.05 \\
\hline Calhoun & 1.09 & 92 & 0.10 \\
\hline Carroll & 3.41 & 88 & 0.30 \\
\hline Chicot & 2.00 & 90 & 0.18 \\
\hline Clark & 0.78 & 89 & 0.07 \\
\hline Clay & 0.00 & 0 & 0.00 \\
\hline Cleburne & 1.00 & 90 & 0.09 \\
\hline Cleveland & 0.80 & 87 & 0.07 \\
\hline Columbia & 0.70 & 86 & 0.06 \\
\hline Conway & 2.00 & 90 & 0.18 \\
\hline Craighead & 5.12 & 90 & 0.46 \\
\hline Crawford & 7.27 & 89 & 0.65 \\
\hline Crittenden & 2.00 & 90 & 0.18 \\
\hline Cross & 0.00 & 0 & 0.00 \\
\hline Dallas & 1.27 & 87 & 0.11 \\
\hline Desha & 0.00 & 0 & 0.00 \\
\hline Drew & 0.75 & 93 & 0.07 \\
\hline Faulkner & 5.29 & 89 & 0.47 \\
\hline Franklin & 0.00 & 0 & 0.00 \\
\hline Fulton & 0.98 & 92 & 0.09 \\
\hline Garland & 0.78 & 90 & 0.07 \\
\hline
\end{tabular}

Department of Commerce, U.S. Census Bureau, 2005) furnish their own water supplies from individual wells; these users are considered self supplied. All self-supplied withdrawals were assumed to be obtained from ground-water sources.

The domestic (self-supplied) water use in each county was computed by multiplying the population (not served by a public-supply system) by a per-capita-use factor (ranging from 80-97 gal/d per person (Halberg, 1977)) (U.S. Department of Commerce, U.S. Census Bureau, 2005). Domestic use in Arkansas for 2005 (table 3) was $18 \mathrm{Mgal} / \mathrm{d}$. Logan County, at $0.86 \mathrm{Mgal} / \mathrm{d}$, had the highest domestic water use in Arkansas in 2005 .

\begin{tabular}{|c|c|c|c|}
\hline County & $\begin{array}{c}\text { Population } \\
\text { (in thousands) }\end{array}$ & $\begin{array}{c}\text { Per capita } \\
\text { use } \\
\text { (gal/d) }\end{array}$ & $\begin{array}{c}\text { Domestic } \\
\text { use } \\
\text { (Mgal/d) }\end{array}$ \\
\hline Grant & 1.38 & 87 & 0.12 \\
\hline Greene & 0.53 & 94 & 0.05 \\
\hline Hempstead & 8.73 & 89 & 0.78 \\
\hline Hot Spring & 1.77 & 91 & 0.16 \\
\hline Howard & 0.54 & 92 & 0.05 \\
\hline Independence & 1.91 & 89 & 0.17 \\
\hline Izard & 1.58 & 88 & 0.14 \\
\hline Jackson & 1.59 & 88 & 0.14 \\
\hline Jefferson & 1.32 & 91 & 0.12 \\
\hline Johnson & 0.00 & 0 & 0.00 \\
\hline Lafayette & 2.17 & 87 & 0.19 \\
\hline Lawrence & 0.00 & 0 & 0.00 \\
\hline Lee & 0.12 & 82 & 0.01 \\
\hline Lincoln & 0.00 & 0 & 0.00 \\
\hline Little River & 1.12 & 90 & 0.10 \\
\hline Logan & 9.70 & 89 & 0.86 \\
\hline Lonoke & 6.15 & 89 & 0.55 \\
\hline Madison & 6.69 & 90 & 0.60 \\
\hline Marion & 6.89 & 89 & 0.61 \\
\hline Miller & 8.14 & 88 & 0.72 \\
\hline Mississippi & 0.00 & 0 & 0.00 \\
\hline Monroe & 0.00 & 0 & 0.00 \\
\hline Montgomery & 5.32 & 88 & 0.47 \\
\hline Nevada & 2.76 & 91 & 0.25 \\
\hline Newton & 0.63 & 95 & 0.06 \\
\hline
\end{tabular}


Table 3. Domestic (self-supplied) water use in Arkansas, 2005.-Continued

[Mgal/d, million gallons per day]

\begin{tabular}{|c|c|c|c|}
\hline County & $\begin{array}{c}\text { Population } \\
\text { (in thousands) }\end{array}$ & $\begin{array}{c}\text { Per capita } \\
\text { use } \\
\text { (gal/d) }\end{array}$ & $\begin{array}{c}\text { Domestic } \\
\text { use } \\
\text { (Mgal/d) }\end{array}$ \\
\hline Ouachita & 0.62 & 97 & 0.06 \\
\hline Perry & 3.57 & 90 & 0.32 \\
\hline Phillips & 0.00 & 0 & 0.00 \\
\hline Pike & 2.62 & 88 & 0.23 \\
\hline Poinsett & 1.81 & 88 & 0.16 \\
\hline Polk & 9.31 & 89 & 0.83 \\
\hline Pope & 3.00 & 90 & 0.27 \\
\hline Prairie & 2.00 & 90 & 0.18 \\
\hline Pulaski & 0.00 & 0 & 0.00 \\
\hline Randolph & 5.46 & 90 & 0.49 \\
\hline Saline & 6.41 & 89 & 0.57 \\
\hline Scott & 4.01 & 90 & 0.36 \\
\hline Searcy & 0.83 & 85 & 0.07 \\
\hline Sebastian & 5.71 & 89 & 0.51 \\
\hline Sevier & 3.00 & 90 & 0.27 \\
\hline Sharp & 3.00 & 90 & 0.27 \\
\hline St. Francis & 2.00 & 90 & 0.18 \\
\hline Stone & 0.00 & 0 & 0.00 \\
\hline Union & 7.41 & 89 & 0.66 \\
\hline Van Buren & 0.50 & 80 & 0.04 \\
\hline Washington & 2.00 & 90 & 0.18 \\
\hline White & 8.20 & 89 & 0.73 \\
\hline Woodruff & 0.94 & 85 & 0.08 \\
\hline Yell & 0.00 & 0 & 0.00 \\
\hline Total & 199.93 & ${ }^{1} 89$ & 17.83 \\
\hline
\end{tabular}

${ }^{1}$ Average per capita use for the State (in gallons per day).

\section{Commercial (self-supplied)}

Commercial water use includes water for motels, hotels, restaurants, office buildings, schools, and civilian and military institutions. Total commercial (self-supplied) water use in Arkansas for 2005 was $27 \mathrm{Mgal} / \mathrm{d}$, of which about 88 percent (24 Mgal/d) was from surface-water and about 12 percent (3.16 Mgal/d) was from ground-water sources (table 4). The largest self-supplied commercial use of water occurred in Lawrence County at $7.94 \mathrm{Mgal} / \mathrm{d}$ of which 97 percent was from surface-water sources. The largest commercial use of ground water (Mississippi River Valley alluvial aquifer) was in Clay County (1.97 Mgal/d).
Table 4. Commercial (self-supplied) water use in Arkansas, 2005.

[Mgal/d, million gallons per day]

\begin{tabular}{|c|c|c|c|}
\hline County & $\begin{array}{c}\text { Ground } \\
\text { water } \\
\text { (Mgal/d) }\end{array}$ & $\begin{array}{c}\text { Surfac } \\
\text { water } \\
\text { (Mgal/d) }\end{array}$ & $\begin{array}{c}\text { Total } \\
\text { (Mgal/d) }\end{array}$ \\
\hline Arkansas & 0.18 & 0.54 & 0.72 \\
\hline Ashley & 0.00 & 0.00 & 0.00 \\
\hline Baxter & 0.00 & 0.00 & 0.00 \\
\hline Benton & 0.00 & 0.15 & 0.15 \\
\hline Boone & 0.00 & 0.00 & 0.00 \\
\hline Bradley & 0.00 & 0.00 & 0.00 \\
\hline Calhoun & 0.00 & 0.00 & 0.00 \\
\hline Carroll & 0.10 & 0.00 & 0.10 \\
\hline Chicot & 0.00 & 0.00 & 0.00 \\
\hline Clark & 0.00 & 0.00 & 0.00 \\
\hline Clay & 1.97 & 0.58 & 2.55 \\
\hline Cleburne & 0.00 & 0.17 & 0.17 \\
\hline Cleveland & 0.00 & 0.00 & 0.00 \\
\hline Columbia & 0.00 & 0.00 & 0.00 \\
\hline Conway & 0.00 & 0.00 & 0.00 \\
\hline Craighead & 0.00 & 0.00 & 0.00 \\
\hline Crawford & 0.00 & 0.00 & 0.00 \\
\hline Crittenden & 0.00 & 0.00 & 0.00 \\
\hline Cross & 0.00 & 0.00 & 0.00 \\
\hline Dallas & 0.00 & 0.00 & 0.00 \\
\hline Desha & 0.00 & 0.00 & 0.00 \\
\hline Drew & 0.00 & 1.25 & 1.25 \\
\hline Faulkner & 0.00 & 0.00 & 0.00 \\
\hline Franklin & 0.00 & 0.00 & 0.00 \\
\hline Fulton & 0.00 & 0.00 & 0.00 \\
\hline Garland & 0.00 & 0.01 & 0.01 \\
\hline Grant & 0.00 & 0.00 & 0.00 \\
\hline Greene & 0.14 & 0.00 & 0.14 \\
\hline Hempstead & 0.01 & 4.82 & 4.83 \\
\hline Hot Spring & 0.00 & 0.00 & 0.00 \\
\hline Howard & 0.00 & 0.00 & 0.00 \\
\hline Independence & 0.00 & 0.00 & 0.00 \\
\hline Izard & 0.00 & 0.00 & 0.00 \\
\hline Jackson & 0.00 & 0.00 & 0.00 \\
\hline Jefferson & 0.00 & 0.67 & 0.67 \\
\hline Johnson & 0.00 & 0.02 & 0.02 \\
\hline Lafayette & 0.00 & 0.00 & 0.00 \\
\hline Lawrence & 0.24 & 7.70 & 7.94 \\
\hline
\end{tabular}


Table 4. Commercial (self-supplied) water use in Arkansas, 2005.-Continued

[Mgal/d, million gallons per day]

\begin{tabular}{|c|c|c|c|}
\hline County & $\begin{array}{c}\text { Ground } \\
\text { water } \\
\text { (Mgal/d) }\end{array}$ & $\begin{array}{c}\text { Surfac } \\
\text { water } \\
\text { (Mgal/d) }\end{array}$ & $\begin{array}{c}\text { Total } \\
(\mathrm{Mgal} / \mathrm{d})\end{array}$ \\
\hline Lee & 0.00 & 0.00 & 0.00 \\
\hline Lincoln & 0.00 & 0.00 & 0.00 \\
\hline Little River & 0.06 & 0.00 & 0.06 \\
\hline Logan & 0.00 & 0.00 & 0.00 \\
\hline Lonoke & 0.00 & 0.00 & 0.00 \\
\hline Madison & 0.00 & 0.00 & 0.00 \\
\hline Marion & 0.00 & 0.00 & 0.00 \\
\hline Miller & 0.00 & 0.80 & 0.80 \\
\hline Mississippi & 0.00 & 0.44 & 0.44 \\
\hline Monroe & 0.00 & 0.00 & 0.00 \\
\hline Montgomery & 0.00 & 0.04 & 0.04 \\
\hline Nevada & 0.00 & 0.00 & 0.00 \\
\hline Newton & 0.00 & 0.00 & 0.00 \\
\hline Ouachita & 0.00 & 0.00 & 0.00 \\
\hline Perry & 0.00 & 0.21 & 0.21 \\
\hline Phillips & 0.00 & 0.00 & 0.00 \\
\hline Pike & 0.00 & 0.03 & 0.03 \\
\hline Poinsett & 0.19 & 0.00 & 0.19 \\
\hline Polk & 0.01 & 0.00 & 0.01 \\
\hline Pope & 0.10 & 0.05 & 0.15 \\
\hline Prairie & 0.00 & 0.00 & 0.00 \\
\hline Pulaski & 0.12 & 0.16 & 0.28 \\
\hline Randolph & 0.00 & 0.00 & 0.00 \\
\hline Saline & 0.00 & 0.00 & 0.00 \\
\hline Scott & 0.00 & 0.00 & 0.00 \\
\hline Searcy & 0.00 & 0.00 & 0.00 \\
\hline Sebastian & 0.00 & 0.00 & 0.00 \\
\hline Sevier & 0.00 & 0.00 & 0.00 \\
\hline Sharp & 0.00 & 0.00 & 0.00 \\
\hline St. Francis & 0.00 & 0.90 & 0.90 \\
\hline Stone & 0.01 & 0.00 & 0.01 \\
\hline Union & 0.00 & 5.46 & 5.46 \\
\hline Van Buren & 0.00 & 0.00 & 0.00 \\
\hline Washington & 0.03 & 0.02 & 0.05 \\
\hline White & 0.00 & 0.00 & 0.00 \\
\hline Woodruff & 0.00 & 0.00 & 0.00 \\
\hline Yell & 0.00 & 0.00 & 0.00 \\
\hline Total & 3.16 & 24.02 & 27.18 \\
\hline
\end{tabular}

\section{Industrial (self-supplied)}

Industrial water use includes water for such purposes as fabrication, processing, washing, and cooling in facilities that manufacture products. The paper products and chemical industries are the largest water users in Arkansas. Total industrial (self-supplied) water use in Arkansas for 2005 was $178 \mathrm{Mgal} / \mathrm{d}$, of which about 37 percent $(66 \mathrm{Mgal} / \mathrm{d})$ was from ground-water and about 63 percent $(113 \mathrm{Mgal} / \mathrm{d})$ was from surface-water sources (table 5). The largest total water use occurred in Ashley County at $59 \mathrm{Mgal} / \mathrm{d}$. The largest ground water use (Sparta-Memphis aquifer) occurred in Jefferson County ( $43 \mathrm{Mgal} / \mathrm{d})$ and the largest surface water use occurred in Ashley County (53 Mgal/d).

Table 5. Industrial (self-supplied) water use in Arkansas, 2005

[Mgal/d, million gallons per day]

\begin{tabular}{|c|c|c|c|}
\hline County & $\begin{array}{c}\text { Ground } \\
\text { water } \\
\text { (Mgal/d) }\end{array}$ & $\begin{array}{c}\text { Surface } \\
\text { water } \\
\text { (Mgal/d) }\end{array}$ & $\begin{array}{c}\text { Total } \\
\text { (Mgal/d) }\end{array}$ \\
\hline Arkansas & 0.00 & 0.00 & 0.00 \\
\hline Ashley & 6.20 & 52.81 & 59.01 \\
\hline Baxter & 0.61 & 0.00 & 0.61 \\
\hline Benton & 0.00 & 0.16 & 0.16 \\
\hline Boone & 0.00 & 0.00 & 0.00 \\
\hline Bradley & 0.46 & 0.00 & 0.46 \\
\hline Calhoun & 0.00 & 0.00 & 0.00 \\
\hline Carroll & 0.00 & 0.00 & 0.00 \\
\hline Chicot & 0.00 & 0.00 & 0.00 \\
\hline Clark & 0.27 & 0.00 & 0.27 \\
\hline Clay & 0.00 & 0.00 & 0.00 \\
\hline Cleburne & 0.00 & 0.00 & 0.00 \\
\hline Cleveland & 0.00 & 0.00 & 0.00 \\
\hline Columbia & 2.08 & 0.09 & 2.17 \\
\hline Conway & 0.00 & 7.62 & 7.62 \\
\hline Craighead & 0.00 & 0.00 & 0.00 \\
\hline Crawford & 0.00 & 0.45 & 0.45 \\
\hline Crittenden & 0.30 & 0.00 & 0.30 \\
\hline Cross & 0.40 & 0.00 & 0.40 \\
\hline Dallas & 0.00 & 0.00 & 0.00 \\
\hline Desha & 3.19 & 9.92 & 13.11 \\
\hline Drew & 0.00 & 0.00 & 0.00 \\
\hline Faulkner & 0.00 & 0.00 & 0.00 \\
\hline Franklin & 0.00 & 0.00 & 0.00 \\
\hline Fulton & 0.00 & 0.00 & 0.00 \\
\hline Garland & 0.06 & 2.35 & 2.41 \\
\hline
\end{tabular}


Table 5. Industrial (self-supplied) water use in Arkansas, 2005.-Continued

$[\mathrm{Mgal} / \mathrm{d}$, million gallons per day]

\begin{tabular}{|c|c|c|c|}
\hline County & $\begin{array}{l}\text { Ground } \\
\text { water } \\
\text { (Mgal/d) }\end{array}$ & $\begin{array}{l}\text { Surface } \\
\text { water } \\
\text { (Mgal/d) }\end{array}$ & $\begin{array}{c}\text { Total } \\
\text { (Mgal/d) }\end{array}$ \\
\hline Grant & 0.15 & 0.00 & 0.15 \\
\hline Greene & 0.00 & 0.00 & 0.00 \\
\hline Hempstead & 0.00 & 0.00 & 0.00 \\
\hline Hot Spring & 0.00 & 0.04 & 0.04 \\
\hline Howard & 0.00 & 1.18 & 1.18 \\
\hline Independence & 0.00 & 32.61 & 32.61 \\
\hline Izard & 0.00 & 2.85 & 2.85 \\
\hline Jackson & 0.00 & 0.00 & 0.00 \\
\hline Jefferson & 42.54 & 0.00 & 42.54 \\
\hline Johnson & 0.00 & 0.00 & 0.00 \\
\hline Lafayette & 0.00 & 0.00 & 0.00 \\
\hline Lawrence & 0.00 & 0.00 & 0.00 \\
\hline Lee & 0.00 & 0.00 & 0.00 \\
\hline Lincoln & 0.00 & 0.00 & 0.00 \\
\hline Little River & 0.24 & 1.31 & 1.55 \\
\hline Logan & 0.00 & 0.00 & 0.00 \\
\hline Lonoke & 0.59 & 0.00 & 0.59 \\
\hline Madison & 0.00 & 0.00 & 0.00 \\
\hline Marion & 0.00 & 0.00 & 0.00 \\
\hline Miller & 0.00 & 0.00 & 0.00 \\
\hline Mississippi & 2.16 & 0.00 & 2.16 \\
\hline Monroe & 0.00 & 0.00 & 0.00 \\
\hline Montgomery & 0.00 & 0.00 & 0.00 \\
\hline Nevada & 0.17 & 0.00 & 0.17 \\
\hline Newton & 0.00 & 0.00 & 0.00 \\
\hline Ouachita & 0.00 & 0.00 & 0.00 \\
\hline Perry & 0.00 & 0.00 & 0.00 \\
\hline Phillips & 0.00 & 0.00 & 0.00 \\
\hline Pike & 0.00 & 0.00 & 0.00 \\
\hline Poinsett & 0.00 & 0.00 & 0.00 \\
\hline Polk & 0.00 & 0.00 & 0.00 \\
\hline Pope & 0.00 & 0.00 & 0.00 \\
\hline Prairie & 0.00 & 0.00 & 0.00 \\
\hline Pulaski & 0.00 & 0.00 & 0.00 \\
\hline Randolph & 0.00 & 0.00 & 0.00 \\
\hline Saline & 0.00 & 0.34 & 0.34 \\
\hline Scott & 0.00 & 0.00 & 0.00 \\
\hline Searcy & 0.00 & 0.00 & 0.00 \\
\hline Sebastian & 0.00 & 0.00 & 0.00 \\
\hline
\end{tabular}

\begin{tabular}{lccc}
\hline \multicolumn{1}{c}{ County } & $\begin{array}{c}\text { Ground } \\
\text { water } \\
\text { (Mgal/d) }\end{array}$ & $\begin{array}{c}\text { Surface } \\
\text { water } \\
\text { (Mgal/d) }\end{array}$ & $\begin{array}{c}\text { Total } \\
\text { (Mgal/d) }\end{array}$ \\
\hline Sevier & 0.00 & 0.34 & 0.34 \\
Sharp & 0.00 & 0.50 & 0.50 \\
St. Francis & 0.00 & 0.00 & 0.00 \\
Stone & 0.00 & 0.00 & 0.00 \\
Union & 6.33 & 0.00 & 6.33 \\
Van Buren & 0.00 & 0.00 & 0.00 \\
Washington & 0.00 & 0.00 & 0.00 \\
White & 0.00 & 0.00 & 0.00 \\
Woodruff & 0.00 & 0.00 & 0.00 \\
Yell & 0.00 & 0.00 & 0.00 \\
& & & \\
Total & 65.75 & 112.57 & 178.32 \\
\hline
\end{tabular}

\section{Mining}

Mining water use includes, in part, water used for coal, sand and gravel washing operations, and saline withdrawals from oil and natural gas production wells. Total mining water use in Arkansas for 2005 was $1.29 \mathrm{Mgal} / \mathrm{d}$, of which about 19 percent $(0.24 \mathrm{Mgal} / \mathrm{d})$ was from ground-water and about 81 percent $(1.05 \mathrm{Mgal} / \mathrm{d}$ ) was from surface-water sources (table 6 ). The largest use of mining water (sand and gravel operations) occurred in Lawrence County at $0.68 \mathrm{Mgal} / \mathrm{d}$ of which 100 percent was from surface-water sources. The largest mining use of ground water (sand and gravel operations) occurred in Craighead and Poinsett Counties at $0.08 \mathrm{Mgal} / \mathrm{d}$ each. 
Table 6. Mining water use in Arkansas, 2005.

[Mgal/d, million gallons per day]

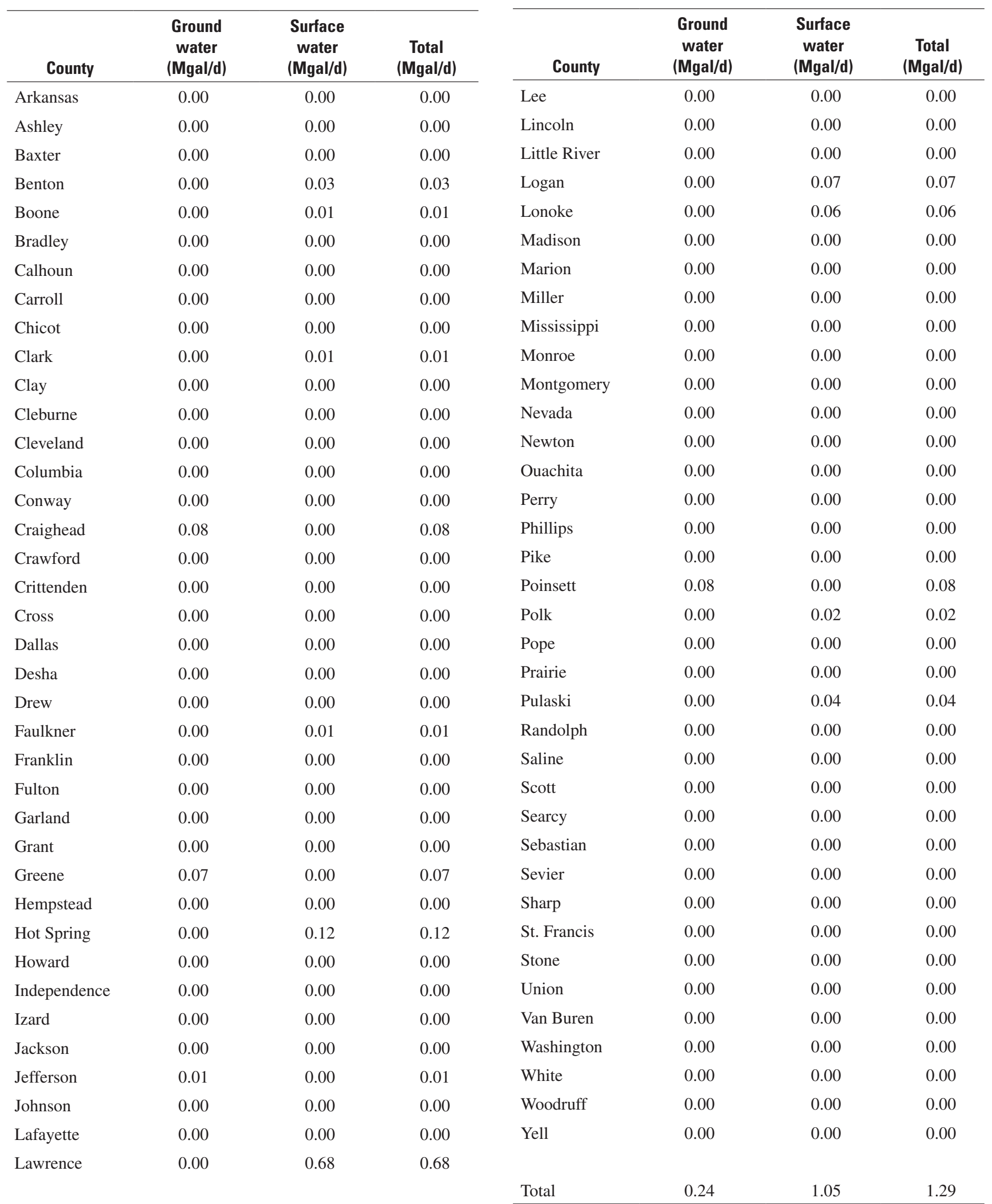




\section{Livestock}

Livestock water use includes water used for stock watering, feed lots, dairy farming, and other farm needs. Many livestock water users fall below the ANRC threshold for reporting either ground water or surface water. Water users that report their usage of water for livestock represent a small portion of the livestock water users in the State. Therefore, the majority of the water use reported for livestock was estimated based on water requirements (Holland, 1987; table 7). The data components required for estimation of water use for livestock include county livestock population and the appropriate water requirement, or water-use coefficient, for each type of livestock produced. Livestock population values for Arkansas for 2005 were published by county by the U.S. Department of Agriculture Crop Reporting Service web site (U.S. Department of Agriculture, 2005). Livestock population was multiplied by the water requirement to obtain the livestock water-use estimate.

Total livestock water use in Arkansas for 2005 was 39 $\mathrm{Mgal} / \mathrm{d}$, of which about 40 percent $(16 \mathrm{Mgal} / \mathrm{d})$ was from ground-water sources and about 60 percent $(23 \mathrm{Mgal} / \mathrm{d})$ was from surface-water sources (table 8). Consumptive use for livestock is considered to be 100 percent of withdrawals. The largest use of livestock water was in Benton County at 2.92 $\mathrm{Mgal} / \mathrm{d}$. Washington County was the largest user of ground water at $1.14 \mathrm{Mgal} / \mathrm{d}$. Benton County was the largest surface water user at $1.80 \mathrm{Mgal} / \mathrm{d}$.

Table 7. Livestock water requirements.

\begin{tabular}{lc}
\hline \multicolumn{1}{c}{ Livestock } & $\begin{array}{c}\text { Water required } \\
\text { per animal } \\
\text { (gallons per day) }\end{array}$ \\
\hline Dairy cattle & 30 \\
Other cattle & 15 \\
Hogs & 2 \\
Poultry & \\
Broilers & 0.04 \\
Hens & 0.06 \\
Turkeys & 0.08 \\
\hline
\end{tabular}

Table 8. Livestock water use in Arkansas, 2005.

[Mgal/d, million gallons per day]

\begin{tabular}{|c|c|c|c|}
\hline County & $\begin{array}{l}\text { Ground } \\
\text { water } \\
\text { (Mgal/d) }\end{array}$ & $\begin{array}{l}\text { Surface } \\
\text { water } \\
\text { (Mgal/d) }\end{array}$ & $\begin{array}{c}\text { Total } \\
\text { (Mgal/d) }\end{array}$ \\
\hline Arkansas & 0.02 & 0.03 & 0.05 \\
\hline Ashley & 0.03 & 0.04 & 0.07 \\
\hline Baxter & 0.15 & 0.21 & 0.36 \\
\hline Benton & 1.12 & 1.80 & 2.92 \\
\hline Boone & 0.54 & 0.81 & 1.35 \\
\hline Bradley & 0.05 & 0.08 & 0.13 \\
\hline Calhoun & 0.02 & 0.04 & 0.06 \\
\hline Carroll & 0.68 & 1.01 & 1.69 \\
\hline Chicot & 0.04 & 0.06 & 0.10 \\
\hline Clark & 0.10 & 0.16 & 0.26 \\
\hline Clay & 0.06 & 0.10 & 0.16 \\
\hline Cleburne & 0.24 & 0.36 & 0.60 \\
\hline Cleveland & 0.12 & 0.20 & 0.32 \\
\hline Columbia & 0.12 & 0.18 & 0.30 \\
\hline Conway & 0.39 & 0.58 & 0.97 \\
\hline Craighead & 0.05 & 0.07 & 0.12 \\
\hline Crawford & 0.24 & 0.35 & 0.59 \\
\hline Crittenden & 0.00 & 0.01 & 0.01 \\
\hline Cross & 0.03 & 0.04 & 0.07 \\
\hline Dallas & 0.02 & 0.03 & 0.05 \\
\hline Desha & 0.02 & 0.03 & 0.05 \\
\hline Drew & 0.07 & 0.09 & 0.16 \\
\hline Faulkner & 0.30 & 0.46 & 0.76 \\
\hline Franklin & 0.40 & 0.60 & 1.00 \\
\hline Fulton & 0.33 & 0.48 & 0.81 \\
\hline Garland & 0.06 & 0.08 & 0.14 \\
\hline Grant & 0.07 & 0.10 & 0.17 \\
\hline Greene & 0.06 & 0.09 & 0.15 \\
\hline Hempstead & 0.58 & 0.85 & 1.43 \\
\hline Hot Spring & 0.11 & 0.17 & 0.28 \\
\hline Howard & 0.44 & 0.65 & 1.09 \\
\hline Independence & 0.35 & 0.53 & 0.88 \\
\hline Izard & 0.24 & 0.37 & 0.61 \\
\hline Jackson & 0.02 & 0.04 & 0.06 \\
\hline Jefferson & 0.04 & 0.05 & 0.09 \\
\hline Johnson & 0.29 & 0.42 & 0.71 \\
\hline Lafayette & 0.26 & 0.39 & 0.65 \\
\hline Lawrence & 0.17 & 0.24 & 0.41 \\
\hline Lee & 0.02 & 0.02 & 0.04 \\
\hline
\end{tabular}


Table 8. Livestock water use in Arkansas, 2005.-Continued

[Mgal/d, million gallons per day]

\begin{tabular}{|c|c|c|c|}
\hline County & $\begin{array}{c}\text { Ground } \\
\text { water } \\
\text { (Mgal/d) }\end{array}$ & $\begin{array}{c}\text { Surface } \\
\text { water } \\
\text { (Mgal/d) }\end{array}$ & $\begin{array}{c}\text { Total } \\
(\mathrm{Mgal} / \mathrm{d})\end{array}$ \\
\hline Lincoln & 0.10 & 0.16 & 0.26 \\
\hline Little River & 0.23 & 0.35 & 0.58 \\
\hline Logan & 0.51 & 0.75 & 1.26 \\
\hline Lonoke & 0.12 & 0.18 & 0.30 \\
\hline Madison & 0.55 & 0.83 & 1.38 \\
\hline Marion & 0.19 & 0.30 & 0.49 \\
\hline Miller & 0.21 & 0.31 & 0.52 \\
\hline Mississippi & 0.00 & 0.01 & 0.01 \\
\hline Monroe & 0.01 & 0.01 & 0.02 \\
\hline Montgomery & 0.20 & 0.29 & 0.49 \\
\hline Nevada & 0.16 & 0.24 & 0.40 \\
\hline Newton & 0.14 & 0.22 & 0.36 \\
\hline Ouachita & 0.05 & 0.07 & 0.12 \\
\hline Perry & 0.13 & 0.19 & 0.32 \\
\hline Phillips & 0.01 & 0.01 & 0.02 \\
\hline Pike & 0.18 & 0.26 & 0.44 \\
\hline Poinsett & 0.02 & 0.03 & 0.05 \\
\hline Polk & 0.33 & 0.48 & 0.81 \\
\hline Pope & 0.38 & 0.57 & 0.95 \\
\hline Prairie & 0.03 & 0.05 & 0.08 \\
\hline Pulaski & 0.06 & 0.10 & 0.16 \\
\hline Randolph & 0.23 & 0.35 & 0.58 \\
\hline Saline & 0.07 & 0.11 & 0.18 \\
\hline Scott & 0.30 & 0.45 & 0.75 \\
\hline Searcy & 0.22 & 0.32 & 0.54 \\
\hline Sebastian & 0.23 & 0.34 & 0.57 \\
\hline Sevier & 0.32 & 0.48 & 0.80 \\
\hline Sharp & 0.24 & 0.37 & 0.61 \\
\hline St. Francis & 0.02 & 0.02 & 0.04 \\
\hline Stone & 0.25 & 0.37 & 0.62 \\
\hline Union & 0.12 & 0.18 & 0.30 \\
\hline Van Buren & 0.16 & 0.24 & 0.40 \\
\hline Washington & 1.14 & 1.71 & 2.85 \\
\hline White & 0.39 & 0.59 & 0.98 \\
\hline Woodruff & 0.01 & 0.01 & 0.02 \\
\hline Yell & 0.37 & 0.55 & 0.92 \\
\hline Total & 15.53 & 23.32 & 38.85 \\
\hline
\end{tabular}

\section{Aquaculture}

Aquaculture water use includes water used for farming of organisms that live in water, such as fish, shellfish, and algae. Total aquaculture water use in Arkansas for 2005 was $256 \mathrm{Mgal} / \mathrm{d}$, of which about 96 percent (246 Mgal/d) was from ground-water sources and about 4 percent (11 Mgal/d) was from surface-water sources (table 9). The largest use of aquacultural water was in Lonoke County at $60 \mathrm{Mgal} / \mathrm{d}$ of which 94 percent was from ground-water sources. The largest aquaculture use of ground water was in Chicot County at $57 \mathrm{Mgal} / \mathrm{d}$ and the largest use of surface water was in Lonoke County at $3.36 \mathrm{Mgal} / \mathrm{d}$. Other counties with notable aquaculture water use (greater than $20 \mathrm{Mgal} / \mathrm{d}$ ) were Prairie and Ashley in order of decreasing use.

Table 9. Aquaculture water use in Arkansas, 2005.

[Mgal/d, million gallons per day]

\begin{tabular}{|c|c|c|c|}
\hline County & $\begin{array}{l}\text { Ground } \\
\text { water } \\
\text { (Mga/d) }\end{array}$ & $\begin{array}{c}\text { Surface } \\
\text { water } \\
\text { (Mgal/d) }\end{array}$ & $\begin{array}{c}\text { Total } \\
\text { (Mgal/d) }\end{array}$ \\
\hline Arkansas & 8.26 & 0.00 & 8.26 \\
\hline Ashley & 22.91 & 0.11 & 23.02 \\
\hline Baxter & 0.00 & 0.00 & 0.00 \\
\hline Benton & 0.07 & 0.00 & 0.07 \\
\hline Boone & 0.00 & 0.00 & 0.00 \\
\hline Bradley & 0.00 & 0.00 & 0.00 \\
\hline Calhoun & 0.00 & 0.00 & 0.00 \\
\hline Carroll & 0.00 & 0.00 & 0.00 \\
\hline Chicot & 56.79 & 0.90 & 57.69 \\
\hline Clark & 0.00 & 0.00 & 0.00 \\
\hline Clay & 1.52 & 0.16 & 1.68 \\
\hline Cleburne & 0.00 & 0.00 & 0.00 \\
\hline Cleveland & 0.00 & 0.00 & 0.00 \\
\hline Columbia & 0.00 & 0.00 & 0.00 \\
\hline Conway & 0.00 & 0.00 & 0.00 \\
\hline Craighead & 0.16 & 0.00 & 0.16 \\
\hline Crawford & 0.00 & 0.00 & 0.00 \\
\hline Crittenden & 1.50 & 0.00 & 1.50 \\
\hline Cross & 0.02 & 0.03 & 0.05 \\
\hline Dallas & 0.00 & 0.00 & 0.00 \\
\hline Desha & 9.67 & 2.61 & 12.28 \\
\hline Drew & 0.03 & 0.00 & 0.03 \\
\hline Faulkner & 0.00 & 0.00 & 0.00 \\
\hline Franklin & 0.00 & 0.00 & 0.00 \\
\hline Fulton & 0.13 & 0.00 & 0.13 \\
\hline Garland & 0.00 & 0.00 & 0.00 \\
\hline
\end{tabular}


Table 9. Aquaculture water use in Arkansas, 2005.-Continued

[Mgal/d, million gallons per day]

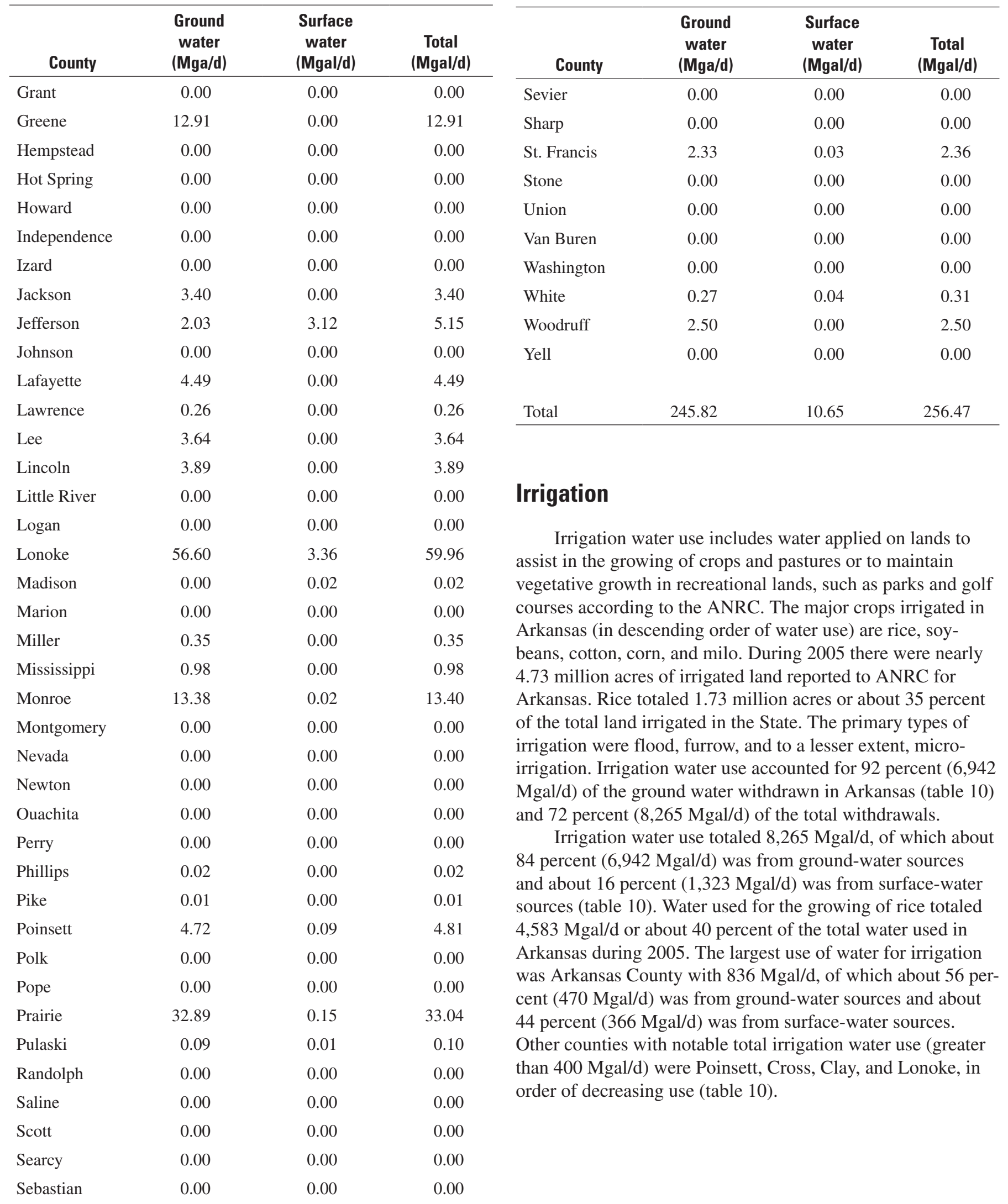


Table 10. Irrigation water use in Arkansas, 2005.

[Mgal/d, million gallons per day]

\begin{tabular}{|c|c|c|c|c|c|c|c|}
\hline \multirow[b]{2}{*}{ County } & \multicolumn{3}{|c|}{$\begin{array}{l}\text { Water withdrawals } \\
\text { (Mgal/d) }\end{array}$} & \multicolumn{4}{|c|}{$\begin{array}{c}\text { Irrigated land } \\
\text { (thousand acres) }\end{array}$} \\
\hline & $\begin{array}{l}\text { Ground } \\
\text { water }\end{array}$ & $\begin{array}{c}\text { Surface } \\
\text { water }\end{array}$ & Total & $\begin{array}{l}\text { Micro- } \\
\text { irrigation }\end{array}$ & Sprinkler & Surface & Total \\
\hline Arkansas & 470.15 & 366.29 & 836.44 & 16.99 & 0.45 & 391.26 & 408.70 \\
\hline Ashley & 124.86 & 12.86 & 137.72 & 0.01 & 0.13 & 105.20 & 105.34 \\
\hline Baxter & 0.00 & 0.00 & 0.00 & 0.00 & 0.00 & 0.00 & 0.00 \\
\hline Benton & 0.07 & 0.08 & 0.15 & 0.13 & 0.09 & 0.10 & 0.32 \\
\hline Boone & 0.00 & 0.04 & 0.04 & 0.06 & 0.00 & 0.00 & 0.06 \\
\hline Bradley & 0.00 & 0.00 & 0.00 & 0.00 & 0.00 & 0.00 & 0.00 \\
\hline Calhoun & 0.01 & 0.00 & 0.01 & 0.00 & 0.02 & 0.00 & 0.02 \\
\hline Carroll & 0.89 & 0.00 & 0.89 & 12.50 & 0.00 & 0.00 & 12.50 \\
\hline Chicot & 190.70 & 69.43 & 260.13 & 3.97 & 26.59 & 142.43 & 172.99 \\
\hline Clark & 0.00 & 3.05 & 3.05 & 1.33 & 0.00 & 1.12 & 2.45 \\
\hline Clay & 466.08 & 9.36 & 475.44 & 0.77 & 10.00 & 189.77 & 200.54 \\
\hline Cleburne & 0.00 & 0.00 & 0.00 & 0.00 & 0.00 & 0.00 & 0.00 \\
\hline Cleveland & 0.00 & 0.00 & 0.00 & 0.00 & 0.00 & 0.00 & 0.00 \\
\hline Columbia & 0.00 & 0.00 & 0.00 & 0.00 & 0.00 & 0.00 & 0.00 \\
\hline Conway & 1.94 & 4.70 & 6.64 & 0.15 & 1.43 & 5.58 & 7.16 \\
\hline Craighead & 350.76 & 44.23 & 394.99 & 0.00 & 7.55 & 250.44 & 257.99 \\
\hline Crawford & 0.05 & 0.99 & 1.04 & 1.43 & 1.39 & 0.70 & 3.52 \\
\hline Crittenden & 148.94 & 0.96 & 149.90 & 0.73 & 59.06 & 62.29 & 122.08 \\
\hline Cross & 596.40 & 44.56 & 640.96 & 0.61 & 8.41 & 228.69 & 237.71 \\
\hline Dallas & 0 & 0.00 & 0 & 0.00 & 0.00 & 0.00 & 0.00 \\
\hline Desha & 284.37 & 87.75 & 372.12 & 0.39 & 2.49 & 214.30 & 217.18 \\
\hline Drew & 74.25 & 12.07 & 86.32 & 0.01 & 0.10 & 54.79 & 54.90 \\
\hline Faulkner & 1.11 & 6.31 & 7.42 & 0.20 & 0.05 & 3.83 & 4.08 \\
\hline Franklin & 0.00 & 0.03 & 0.03 & 0.10 & 0.00 & 0.00 & 0.10 \\
\hline Fulton & 0.13 & 0.00 & 0.13 & 0.00 & 0.00 & 0.18 & 0.18 \\
\hline Garland & 0.00 & 0.57 & 0.57 & 0.00 & 0.02 & 0.10 & 0.12 \\
\hline Grant & 0.23 & 0.00 & 0.23 & 0.00 & 0.00 & 0.09 & 0.09 \\
\hline Greene & 206.17 & 3.81 & 209.98 & 0.00 & 6.11 & 142.27 & 148.38 \\
\hline Hempstead & 0.00 & 0.00 & 0.00 & 0.00 & 0.00 & 0.00 & 0.00 \\
\hline Hot Spring & 0.00 & 6.86 & 6.86 & 0.00 & 0.00 & 3.01 & 3.01 \\
\hline Howard & 0.00 & 0.00 & 0.00 & 0.00 & 0.00 & 0.00 & 0.00 \\
\hline Independence & 40.43 & 14.97 & 55.40 & 0.47 & 6.45 & 22.56 & 29.48 \\
\hline Izard & 0.40 & 0.00 & 0.40 & 0.00 & 0.00 & 0.20 & 0.20 \\
\hline Jackson & 369.86 & 17.54 & 387.40 & 8.25 & 19.75 & 189.57 & 217.57 \\
\hline Jefferson & 215.58 & 43.01 & 258.59 & 0.55 & 4.13 & 171.84 & 176.52 \\
\hline Johnson & 0.03 & 0.00 & 0.03 & 0.00 & 0.01 & 0.58 & 0.59 \\
\hline Lafayette & 28.42 & 1.01 & 29.43 & 0.16 & 2.73 & 18.31 & 21.20 \\
\hline Lawrence & 220.77 & 24.21 & 244.98 & 0.05 & 1.26 & 134.94 & 136.25 \\
\hline
\end{tabular}


Table 10. Irrigation water use in Arkansas, 2005.-Continued

[Mgal/d, million gallons per day]

\begin{tabular}{|c|c|c|c|c|c|c|c|}
\hline \multirow[b]{2}{*}{ County } & \multicolumn{3}{|c|}{$\begin{array}{l}\text { Water withdrawals } \\
\text { (Mgal/d) }\end{array}$} & \multicolumn{4}{|c|}{$\begin{array}{l}\text { Irrigated land } \\
\text { (thousand acres) }\end{array}$} \\
\hline & $\begin{array}{l}\text { Ground } \\
\text { water }\end{array}$ & $\begin{array}{l}\text { Surface } \\
\text { water }\end{array}$ & Total & $\begin{array}{l}\text { Micro- } \\
\text { irrigation }\end{array}$ & Sprinkler & Surface & Total \\
\hline Lee & 254.71 & 4.85 & 259.56 & 3.33 & 27.84 & 132.47 & 163.64 \\
\hline Lincoln & 177.81 & 23.34 & 201.15 & 1.35 & 0.00 & 112.37 & 113.72 \\
\hline Little River & 3.19 & 5.15 & 8.34 & 0.20 & 0.00 & 5.40 & 5.60 \\
\hline Logan & 0.28 & 0.07 & 0.35 & 0.00 & 0.00 & 0.60 & 0.60 \\
\hline Lonoke & 355.84 & 103.56 & 459.40 & 1.94 & 2.21 & 264.50 & 268.65 \\
\hline Madison & 0.00 & 0.13 & 0.13 & 0.04 & 0.08 & 0.02 & 0.14 \\
\hline Marion & 0.00 & 0.00 & 0.00 & 0.00 & 0.00 & 0.00 & 0.00 \\
\hline Miller & 9.75 & 67.99 & 77.74 & 0.24 & 2.78 & 30.78 & 33.80 \\
\hline Mississippi & 270.57 & 2.12 & 272.69 & 7.23 & 126.22 & 119.11 & 252.56 \\
\hline Monroe & 269.62 & 22.56 & 292.18 & 0.26 & 1.59 & 166.31 & 168.16 \\
\hline Montgomery & 0.00 & 0.01 & 0.01 & 0.00 & 0.01 & 0.00 & 0.01 \\
\hline Nevada & 0.00 & 0.35 & 0.35 & 0.00 & 0.14 & 0.14 & 0.28 \\
\hline Newton & 0.00 & 0.01 & 0.01 & 0.00 & 0.00 & 0.00 & 0.00 \\
\hline Ouachita & 0.00 & 0.00 & 0.00 & 0.00 & 0.00 & 0.00 & 0.00 \\
\hline Perry & 0.00 & 13.17 & 13.17 & 0.67 & 1.45 & 2.46 & 4.58 \\
\hline Phillips & 204.31 & 1.77 & 206.08 & 8.57 & 75.55 & 96.18 & 180.30 \\
\hline Pike & 0.01 & 0.07 & 0.08 & 0.01 & 0.08 & 0.00 & 0.09 \\
\hline Poinsett & 671.27 & 83.69 & 754.96 & 6.45 & 33.02 & 287.03 & 326.50 \\
\hline Polk & 0.00 & 0.15 & 0.15 & 0.00 & 0.17 & 0.00 & 0.17 \\
\hline Pope & 1.34 & 0.61 & 1.95 & 0.12 & 0.24 & 1.21 & 1.57 \\
\hline Prairie & 218.68 & 89.64 & 308.32 & 3.77 & 0.85 & 200.80 & 205.42 \\
\hline Pulaski & 20.76 & 12.61 & 33.37 & 0.09 & 2.67 & 22.79 & 25.55 \\
\hline Randolph & 101.46 & 37.38 & 138.84 & 0.77 & 0.29 & 59.20 & 60.26 \\
\hline Saline & 0.02 & 0.60 & 0.62 & 0.10 & 0.29 & 0.00 & 0.39 \\
\hline Scott & 0.00 & 0.00 & 0.00 & 0.00 & 0.00 & 0.00 & 0.00 \\
\hline Searcy & 0.00 & 0.00 & & 0.00 & 0.00 & 0.00 & 0.00 \\
\hline Sebastian & 0.00 & 0.08 & 0.08 & 0.00 & 0.01 & 0.06 & 0.07 \\
\hline Sevier & 0.00 & 0.00 & 0.00 & 0.01 & 0.00 & 0.00 & 0.01 \\
\hline Sharp & 0.00 & 0.11 & 0.11 & 0.13 & 0.04 & 0.07 & 0.24 \\
\hline St. Francis & 285.34 & 17.34 & 302.68 & 0.00 & 29.97 & 135.93 & 165.90 \\
\hline Stone & 0.32 & 0.00 & 0.32 & 0.00 & 0.22 & 0.06 & 0.28 \\
\hline Union & 0.00 & 0.00 & 0.00 & 0.00 & 0.00 & 0.00 & 0.00 \\
\hline Van Buren & 0.21 & 0.00 & 0.21 & 0.00 & 0.00 & 0.12 & 0.12 \\
\hline Washington & 0.00 & 0.19 & 0.19 & 0.06 & 0.12 & 0.04 & 0.22 \\
\hline White & 41.57 & 33.84 & 75.41 & 0.40 & 2.93 & 37.32 & 40.65 \\
\hline Woodruff & 262.50 & 24.60 & 287.10 & 0.25 & 15.37 & 152.61 & 168.23 \\
\hline Yell & 0.00 & 2.59 & 2.59 & 0.00 & 0.00 & 1.08 & 1.08 \\
\hline Total & $6,942.16$ & $1,323.27$ & $8,265.12$ & 84.85 & 482.36 & $4,162.81$ & $4,730.02$ \\
\hline
\end{tabular}




\section{Duck (Hunting) Clubs}

Duck (hunting) clubs are for the restricted use of their members and their guests. Both ground and surface water are applied to fill open fields or large bayous or ponds with water to attract migratory waterfowl for the purpose of hunting. Until recently (2003), duck (hunting) club water use had been included in the irrigation category. Water-use accounting by duck clubs is important because duck hunting season (usually between October and January) is when the greatest groundwater recharge occurs. Total duck (hunting) club water use in Arkansas for 2005 was $269 \mathrm{Mgal} / \mathrm{d}$, of which about 30 percent $(81 \mathrm{Mgal} / \mathrm{d})$ was from ground-water and about 70 percent $(188$ $\mathrm{Mgal} / \mathrm{d}$ ) was from surface-water sources (table 11). The largest use of water for duck (hunting) clubs was in Arkansas County at $115 \mathrm{Mgal} / \mathrm{d}$ of which 79 percent $(91 \mathrm{Mgal} / \mathrm{d})$ was from surface-water sources and 21 percent $(25 \mathrm{Mgal} / \mathrm{d})$ was from ground-water sources. Other counties with substantial duck (hunting) club water use (greater than $10 \mathrm{Mgal} / \mathrm{d}$ ) were Miller, Desha, Prairie, Jackson, and Jefferson, in order of decreasing use.

Table 11. Duck (hunting) clubs water use in Arkansas, 2005.

[Mgal/d, million gallons per day]

\begin{tabular}{|c|c|c|c|}
\hline County & $\begin{array}{c}\text { Ground } \\
\text { water } \\
\text { (Mgal/d) }\end{array}$ & $\begin{array}{c}\text { Surface } \\
\text { water } \\
\text { (Mgal/d) }\end{array}$ & $\begin{array}{c}\text { Total } \\
\text { (Mgal/d) }\end{array}$ \\
\hline Arkansas & 24.67 & 90.78 & 115.45 \\
\hline Ashley & 0.00 & 0.00 & 0.00 \\
\hline Baxter & 0.00 & 0.00 & 0.00 \\
\hline Benton & 0.00 & 0.00 & 0.00 \\
\hline Boone & 0.00 & 0.00 & 0.00 \\
\hline Bradley & 0.00 & 0.00 & 0.00 \\
\hline Calhoun & 0.00 & 0.00 & 0.00 \\
\hline Carroll & 0.00 & 0.00 & 0.00 \\
\hline Chicot & 0.00 & 0.00 & 0.00 \\
\hline Clark & 0.00 & 0.03 & 0.03 \\
\hline Clay & 0.00 & 0.00 & 0.00 \\
\hline Cleburne & 0.00 & 0.00 & 0.00 \\
\hline Cleveland & 0.00 & 0.00 & 0.00 \\
\hline Columbia & 0.00 & 0.00 & 0.00 \\
\hline Conway & 0.00 & 0.00 & 0.00 \\
\hline Craighead & 0.00 & 0.38 & 0.38 \\
\hline Crawford & 0.00 & 0.00 & 0.00 \\
\hline Crittenden & 0.12 & 0.00 & 0.12 \\
\hline Cross & 0.00 & 0.38 & 0.38 \\
\hline Dallas & 0.31 & 0.00 & 0.31 \\
\hline Desha & 4.91 & 8.96 & 13.87 \\
\hline
\end{tabular}

Table 11. Duck (hunting) clubs water use in Arkansas, 2005.-Continued

[Mgal/d, million gallons per day]

\begin{tabular}{|c|c|c|c|}
\hline County & $\begin{array}{c}\text { Ground } \\
\text { water } \\
\text { (Mgal/d) }\end{array}$ & $\begin{array}{c}\text { Surface } \\
\text { water } \\
\text { (Mgal/d) }\end{array}$ & $\begin{array}{c}\text { Total } \\
\text { (Mgal/d) }\end{array}$ \\
\hline Drew & 0.20 & 0.00 & 0.20 \\
\hline Faulkner & 0.00 & 0.00 & 0.00 \\
\hline Franklin & 0.00 & 0.00 & 0.00 \\
\hline Fulton & 0.00 & 0.00 & 0.00 \\
\hline Garland & 0.00 & 0.00 & 0.00 \\
\hline Grant & 0.00 & 0.00 & 0.00 \\
\hline Greene & 0.00 & 0.00 & 0.00 \\
\hline Hempstead & 0.00 & 2.24 & 2.24 \\
\hline Hot Spring & 0.00 & 0.43 & 0.43 \\
\hline Howard & 0.00 & 0.00 & 0.00 \\
\hline Independence & 0.02 & 0.45 & 0.47 \\
\hline Izard & 0.00 & 0.00 & 0.00 \\
\hline Jackson & 8.18 & 5.00 & 13.18 \\
\hline Jefferson & 5.14 & 7.91 & 13.05 \\
\hline Johnson & 0.00 & 0.00 & 0.00 \\
\hline Lafayette & 4.62 & 0.41 & 5.03 \\
\hline Lawrence & 0.22 & 0.00 & 0.22 \\
\hline Lee & 7.42 & 0.54 & 7.96 \\
\hline Lincoln & 0.03 & 0.03 & 0.06 \\
\hline Little River & 0.00 & 2.28 & 2.28 \\
\hline Logan & 0.00 & 0.00 & 0.00 \\
\hline Lonoke & 3.65 & 3.25 & 6.90 \\
\hline Madison & 0.00 & 0.00 & 0.00 \\
\hline Marion & 0.00 & 0.00 & 0.00 \\
\hline Miller & 5.00 & 41.20 & 46.20 \\
\hline Mississippi & 0.00 & 0.00 & 0.00 \\
\hline Monroe & 5.54 & 3.82 & 9.36 \\
\hline Montgomery & 0.00 & 0.00 & 0.00 \\
\hline Nevada & 0.00 & 0.00 & 0.00 \\
\hline Newton & 0.00 & 0.00 & 0.00 \\
\hline Ouachita & 0.00 & 0.00 & 0.00 \\
\hline Perry & 0.00 & 0.00 & 0.00 \\
\hline Phillips & 0.04 & 0.00 & 0.04 \\
\hline Pike & 0.00 & 0.00 & 0.00 \\
\hline Poinsett & 0.75 & 6.67 & 7.42 \\
\hline Polk & 0.00 & 0.00 & 0.00 \\
\hline Pope & 0.00 & 0.00 & 0.00 \\
\hline Prairie & 1.01 & 12.34 & 13.35 \\
\hline
\end{tabular}


Table 11. Duck (hunting) clubs water use in Arkansas, 2005.-Continued

[Mgal/d, million gallons per day]

\begin{tabular}{lccr}
\hline \multicolumn{1}{c}{ County } & $\begin{array}{c}\text { Ground } \\
\text { water } \\
\text { (Mgal/d) }\end{array}$ & $\begin{array}{c}\text { Surface } \\
\text { water } \\
\text { (Mgal/d) }\end{array}$ & $\begin{array}{c}\text { Total } \\
\text { (Mgal/d) }\end{array}$ \\
\hline Pulaski & 0.80 & 0.09 & 0.89 \\
Randolph & 0.00 & 0.00 & 0.00 \\
Saline & 0.00 & 0.00 & 0.00 \\
Scott & 0.00 & 0.00 & 0.00 \\
Searcy & 0.00 & 0.00 & 0.00 \\
Sebastian & 0.00 & 0.00 & 0.00 \\
Sevier & 0.00 & 0.00 & 0.00 \\
Sharp & 0.00 & 0.00 & 0.00 \\
St Francis & 2.68 & 0.28 & 2.96 \\
Stone & 0.00 & 0.00 & 0.00 \\
Union & 0.00 & 0.00 & 0.00 \\
Van Buren & 0.00 & 0.00 & 0.00 \\
Washington & 0.00 & 0.00 & 0.00 \\
White & 4.88 & 0.19 & 5.07 \\
Woodruff & 0.95 & 0.27 & 1.22 \\
Yell & 0.00 & 0.00 & 0.00 \\
Total & 81.14 & 187.93 & 269.07 \\
\hline
\end{tabular}

\section{Thermoelectric Power Generation}

Water is used in the process of the generation of thermoelectric power for boiler makeup, cooling, and domestic purposes. Surface water generally is diverted for "once through" cooling and then returned to a stream. Total thermoelectric power generation water use in Arkansas for 2005 was $1,997 \mathrm{Mgal} / \mathrm{d}$ of which more than 99 percent $(1,996 \mathrm{Mgal} / \mathrm{d})$ was from surface-water sources and less than 1 percent $(0.93$ $\mathrm{Mgal} / \mathrm{d}$ ) was from ground-water sources (table 12). Water used for thermoelectric power generation was about 17 percent of the total water used in Arkansas during 2005 and 51 percent of the total surface water used. About 58 percent $(1,152 \mathrm{Mgal} / \mathrm{d})$ of the water used in this category was used for cooling at a nuclear power plant in Pope County. About 98 percent $(1,957$ $\mathrm{Mgal} / \mathrm{d}$ ) of the water used in this category was used in Pope (Lake Dardanelle), Benton, Hot Spring (Lake Catherine and Ouachita River), Woodruff (White River), and Ouachita (Ouachita River) Counties, in order of decreasing use. 
Table 12. Thermoelectric-power generation water use in Arkansas, 2005.

\begin{tabular}{|c|c|c|c|c|c|c|c|}
\hline County & $\begin{array}{c}\text { Ground water } \\
\text { (Mgal/d) }\end{array}$ & $\begin{array}{c}\text { Surface water } \\
\text { (Mgal/d) }\end{array}$ & $\begin{array}{c}\text { Total } \\
\text { (Mgal/d) }\end{array}$ & County & $\begin{array}{l}\text { Ground water } \\
\text { (Mgal/d) }\end{array}$ & $\begin{array}{c}\text { Surface water } \\
\text { (Mgal/d) }\end{array}$ & $\begin{array}{c}\text { Total } \\
\text { (Mgal/d) }\end{array}$ \\
\hline Arkansas & 0.00 & 0.00 & 0.00 & Lee & 0.00 & 0.00 & 0.00 \\
\hline Ashley & 0.00 & 10.15 & 10.15 & Lincoln & 0.00 & 0.00 & 0.00 \\
\hline Baxter & 0.00 & 0.00 & 0.00 & Little River & 0.00 & 0.00 & 0.00 \\
\hline Benton & 0.00 & 377.97 & 377.97 & Logan & 0.00 & 0.00 & 0.00 \\
\hline Boone & 0.00 & 0.00 & 0.00 & Lonoke & 0.00 & 0.00 & 0.00 \\
\hline Bradley & 0.00 & 0.00 & 0.00 & Madison & 0.00 & 0.00 & 0.00 \\
\hline Calhoun & 0.00 & 0.00 & 0.00 & Marion & 0.00 & 0.00 & 0.00 \\
\hline Carroll & 0.00 & 0.00 & 0.00 & Miller & 0.00 & 0.00 & 0.00 \\
\hline Chicot & 0.00 & 0.00 & 0.00 & Mississippi & 0.00 & 0.00 & 0.00 \\
\hline Clark & 0.00 & 0.00 & 0.00 & Monroe & 0.00 & 0.00 & 0.00 \\
\hline Clay & 0.00 & 0.00 & 0.00 & Montgomery & 0.00 & 0.00 & 0.00 \\
\hline Cleburne & 0.00 & 0.00 & 0.00 & Nevada & 0.00 & 0.00 & 0.00 \\
\hline Cleveland & 0.00 & 0.00 & 0.00 & Newton & 0.00 & 0.00 & 0.00 \\
\hline Columbia & 0.00 & 0.00 & 0.00 & Ouachita & 0.00 & 55.58 & 55.58 \\
\hline Conway & 0.00 & 0.00 & 0.00 & Perry & 0.00 & 0.00 & 0.00 \\
\hline Craighead & 0.00 & 0.00 & 0.00 & Phillips & 0.43 & 0.00 & 0.43 \\
\hline Crawford & 0.00 & 0.00 & 0.00 & Pike & 0.00 & 0.00 & 0.00 \\
\hline Crittenden & 0.00 & 0.00 & 0.00 & Poinsett & 0.00 & 0.00 & 0.00 \\
\hline Cross & 0.00 & 0.00 & 0.00 & Polk & 0.00 & 0.00 & 0.00 \\
\hline Dallas & 0.00 & 0.00 & 0.00 & Pope & 0.00 & $1,152.00$ & $1,152.00$ \\
\hline Desha & 0.00 & 0.00 & 0.00 & Prairie & 0.00 & 0.00 & 0.00 \\
\hline Drew & 0.00 & 0.00 & 0.00 & Pulaski & 0.00 & 0.20 & 0.20 \\
\hline Faulkner & 0.00 & 0.00 & 0.00 & Randolph & 0.00 & 0.00 & 0.00 \\
\hline Franklin & 0.00 & 0.00 & 0.00 & Saline & 0.00 & 0.00 & 0.00 \\
\hline Fulton & 0.00 & 0.00 & 0.00 & Scott & 0.00 & 0.00 & 0.00 \\
\hline Garland & 0.00 & 0.00 & 0.00 & Searcy & 0.00 & 0.00 & 0.00 \\
\hline Grant & 0.00 & 0.00 & 0.00 & Sebastian & 0.00 & 0.00 & 0.00 \\
\hline Greene & 0.00 & 0.00 & 0.00 & Sevier & 0.00 & 0.00 & 0.00 \\
\hline Hempstead & 0.00 & 0.00 & 0.00 & Sharp & 0.00 & 0.00 & 0.00 \\
\hline Hot Spring & 0.00 & 311.64 & 311.64 & St. Francis & 0.00 & 0.00 & 0.00 \\
\hline Howard & 0.00 & 0.00 & 0.00 & Stone & 0.00 & 0.00 & 0.00 \\
\hline Independence & 0.00 & 8.66 & 8.66 & Union & 0.00 & 5.01 & 5.01 \\
\hline Izard & 0.00 & 0.00 & 0.00 & Van Buren & 0.00 & 0.00 & 0.00 \\
\hline Jackson & 0.00 & 0.00 & 0.00 & Washington & 0.00 & 0.00 & 0.00 \\
\hline Jefferson & 0.00 & 15.51 & 15.51 & White & 0.00 & 0.00 & 0.00 \\
\hline Johnson & 0.00 & 0.00 & 0.00 & Woodruff & 0.00 & 59.59 & 59.59 \\
\hline Lafayette & 0.50 & 0.00 & 0.50 & Yell & 0.00 & 0.00 & 0.00 \\
\hline \multirow[t]{2}{*}{ Lawrence } & 0.00 & 0.00 & 0.00 & & & & \\
\hline & & & & Total & 0.93 & $1,996.31$ & $1,997.24$ \\
\hline
\end{tabular}




\section{Changes in Water Use, 1965-2005}

Total water use in Arkansas has increased about 435 percent between 1965 and 2005 (table 13). Total groundwater use in Arkansas between 1965 and 2005 has increased from 1,231 to $7,510 \mathrm{Mgal} / \mathrm{d}$, an increase of about 510 percent. Total surface-water use in Arkansas between 1965 and 2005 increased from 911 to 3,945 Mgal/d, an increase of 333 percent. Ground-water irrigation use between 1965 and 2005 increased from 949 to $6,942 \mathrm{Mgal} / \mathrm{d}$, an increase of about 632 percent. Surface-water irrigation use between 1965 and 2005 increased from 211 to $1,323 \mathrm{Mgal} / \mathrm{d}$, an increase of about 527 percent (table 13, fig. 9). Public supply water use between 1965 and 2005 increased from 127 to $404 \mathrm{Mgal} / \mathrm{d}$, an increase of 218 percent (table 13). As the population served by public supply (Arkansas Department of Health, 2007) between 1965 and 2005 increased 36 percent from approximately 1.9 to 2.6 million people (fig. 10), the per capita use of public supply water increased about 35 percent from approximately 116 to $157 \mathrm{gal} / \mathrm{d}$.

Table 13. Changes in water use for selected categories in Arkansas, 1965-2005.

[Mgal/d, million gallons per day]

\begin{tabular}{|c|c|c|c|c|c|c|c|c|c|}
\hline \multirow[b]{2}{*}{ Year } & \multicolumn{2}{|c|}{ Irrigation } & \multicolumn{4}{|c|}{ Public supply } & \multicolumn{3}{|c|}{ Total } \\
\hline & $\begin{array}{l}\text { Ground } \\
\text { water } \\
\text { (Mgal/d) }\end{array}$ & $\begin{array}{l}\text { Surface } \\
\text { water } \\
\text { (Mgal/d) }\end{array}$ & $\begin{array}{c}\text { Population } \\
\text { served }\end{array}$ & $\begin{array}{l}\text { Ground } \\
\text { water } \\
\text { (Mgal/d) }\end{array}$ & $\begin{array}{l}\text { Surface } \\
\text { water } \\
\text { (Mgal/d) }\end{array}$ & $\begin{array}{c}\text { Per capita } \\
\text { use } \\
\text { (gallon/ } \\
\text { day/person) }\end{array}$ & $\begin{array}{l}\text { Ground } \\
\text { water } \\
\text { (Mgal/d) }\end{array}$ & $\begin{array}{l}\text { Surface } \\
\text { water } \\
\text { (Mgal/d) }\end{array}$ & $\begin{array}{c}\text { Total } \\
\text { (Mgal/d) }\end{array}$ \\
\hline 1965 & 949 & 211 & $1,900,000$ & 54 & 73 & 116 & 1,231 & 911 & 2,142 \\
\hline 1970 & 1,064 & 229 & $1,920,000$ & 71 & 95 & 135 & 1,530 & 1,531 & 3,061 \\
\hline 1975 & 2,094 & 346 & $2,116,000$ & 89 & 118 & 180 & 2,596 & 2,468 & 5,064 \\
\hline 1980 & 3,484 & 597 & $2,283,733$ & 110 & 153 & 155 & 4,053 & 2,166 & 6,219 \\
\hline 1985 & 3,330 & 541 & $2,358,000$ & 104 & 156 & 153 & 3,810 & 2,041 & 5,851 \\
\hline 1990 & 4,296 & 949 & $2,353,000$ & 119 & 190 & 173 & 4,708 & 3,128 & 7,836 \\
\hline 1995 & 4,925 & 1,013 & $2,484,000$ & 135 & 246 & 191 & 5,456 & 3,311 & 8,767 \\
\hline 2000 & 6,506 & 1,407 & $2,322,520$ & 138 & 289 & 181 & 6,952 & 4,011 & 10,963 \\
\hline 2005 & 6,942 & 1,323 & $2,579,200$ & 138 & 266 & 157 & 7,510 & 3,945 & 11,455 \\
\hline
\end{tabular}

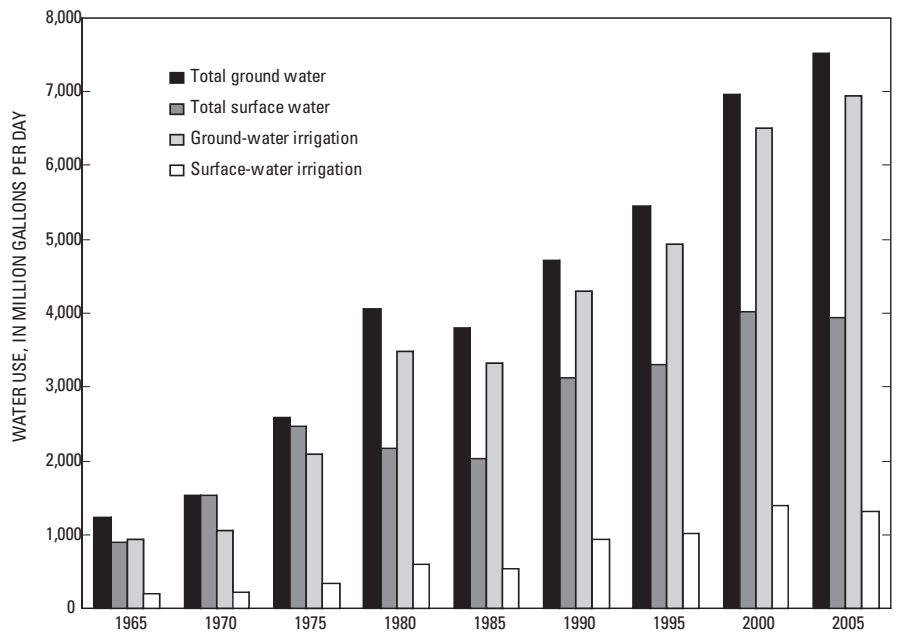

Figure 9. Total ground- and surface-water use compared to irrigation water use in Arkansas, 1965-2005.

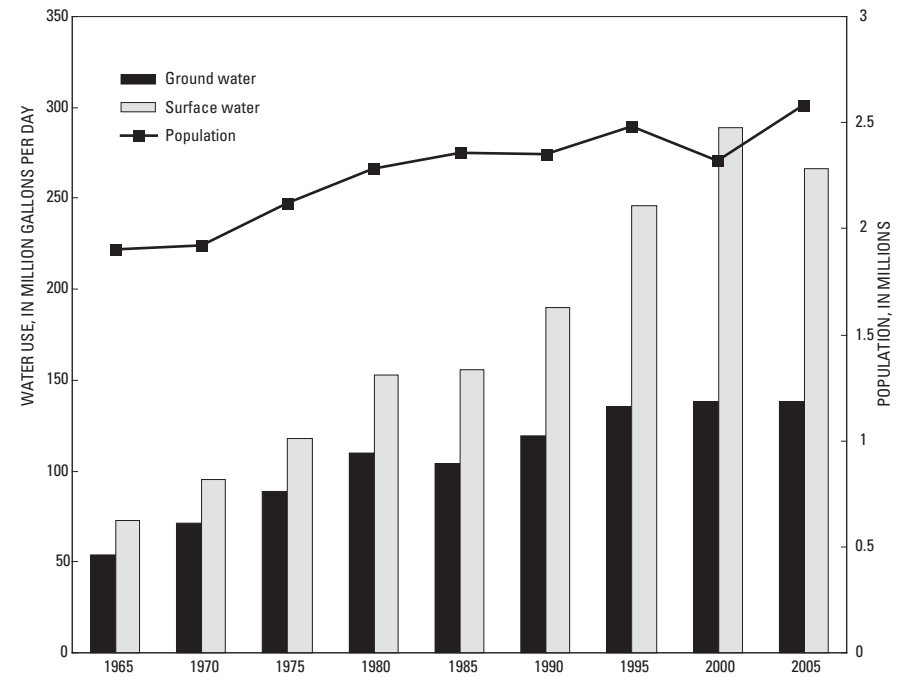

Figure 10. Ground- and surface-water use and population served for public-supply systems in Arkansas, 1965-2005. 


\section{Sources of Ground-Water Withdrawals}

Ground-water withdrawals comprised about 66 percent $(7,510 \mathrm{Mgal} / \mathrm{d})$ of the total amount of water used in Arkansas in 2005 (table 13). About 98 percent (7,349 Mgal/d) of the water was withdrawn from the Mississippi River Valley alluvial aquifer (table 14) and the Sparta-Memphis aquifer (table 14, fig. 11).

The Mississippi River Valley alluvial aquifer supplied about 96 percent $(7,180 \mathrm{Mgal} / \mathrm{d})$ of all ground water used in Arkansas in 2005 (table 14). Poinsett, Cross, and Arkansas were the counties with the most use from the Mississippi River Valley alluvial aquifer.
The Sparta-Memphis aquifer supplied about 2 percent of the ground water used in Arkansas, mostly by industry and public-supply systems in eastern and southern Arkansas. The counties with the highest use from the Sparta-Memphis aquifer are Jefferson and Arkansas Counties using about 51 percent of the total water withdrawn from the aquifer.
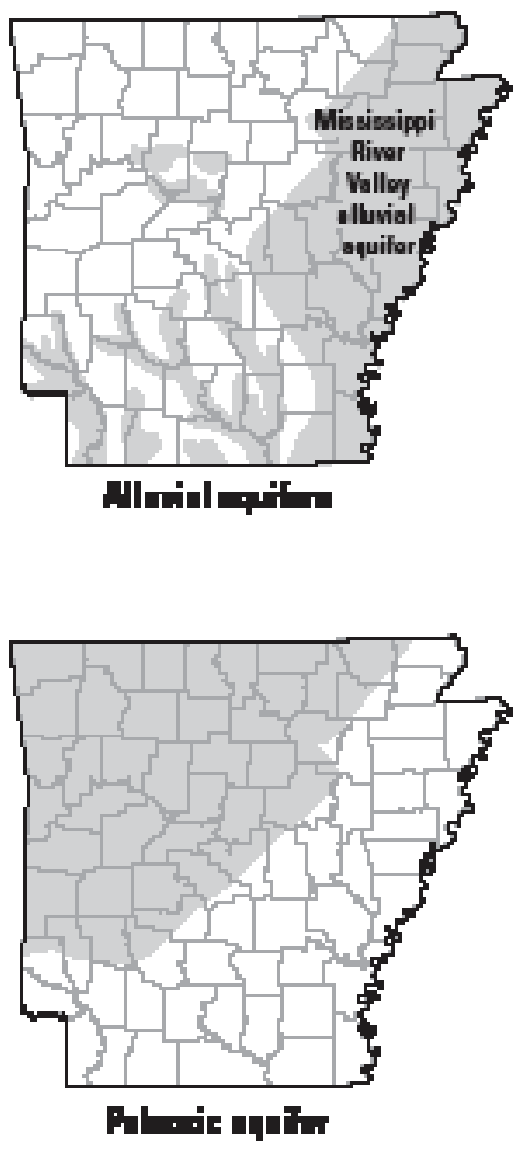
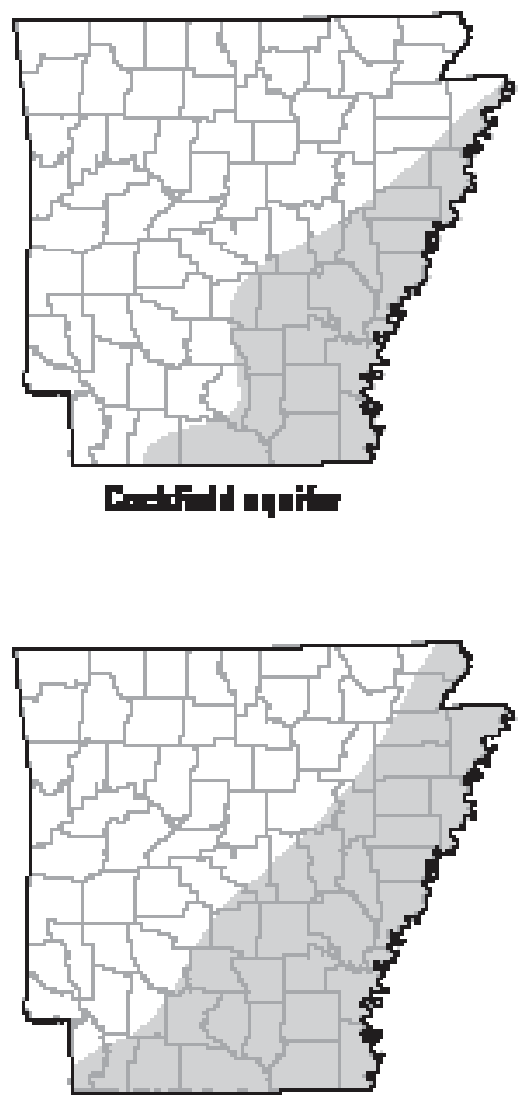

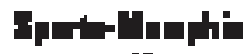

rive
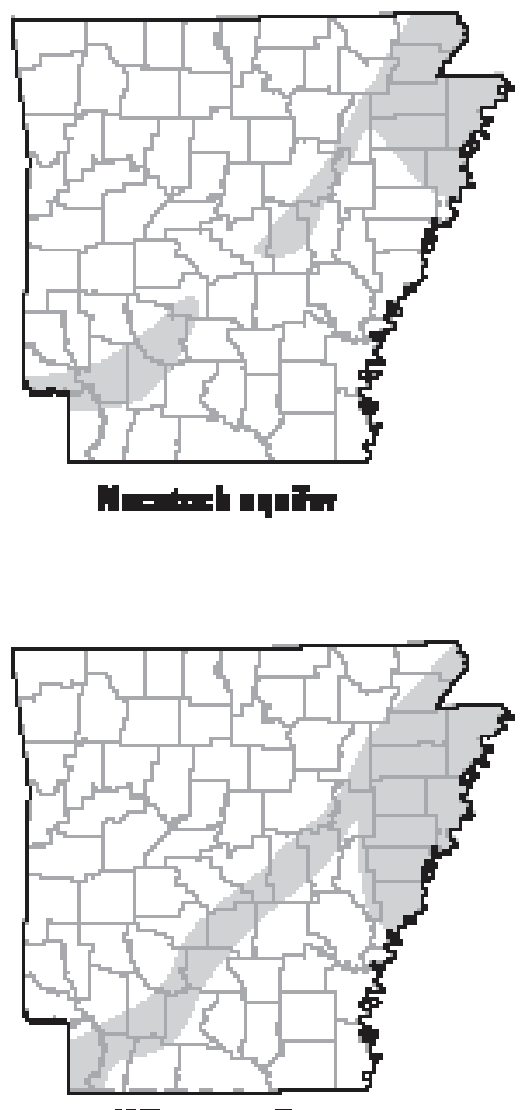

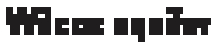

Figure 11. Location of selected major aquifers in Arkansas. 


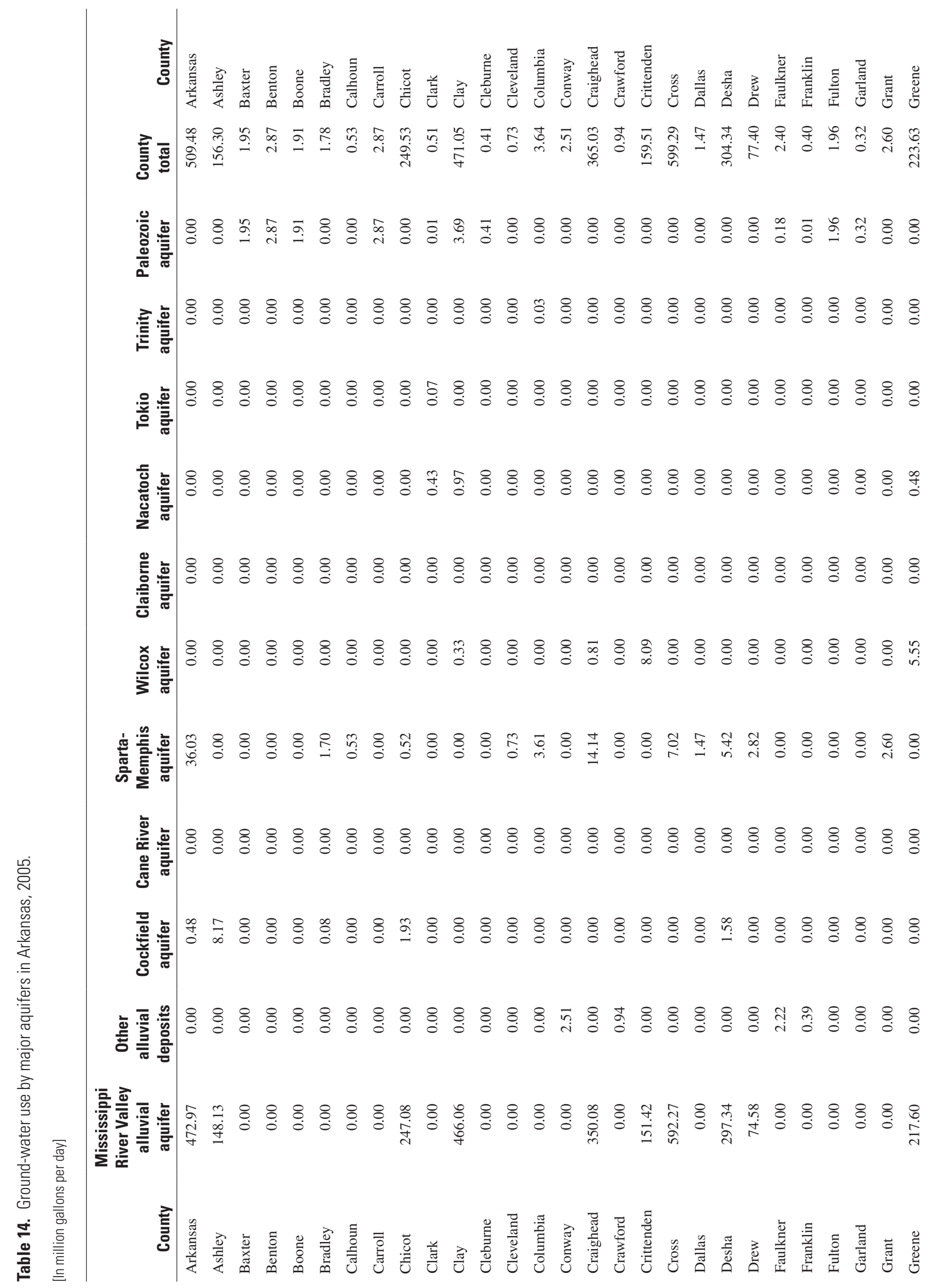




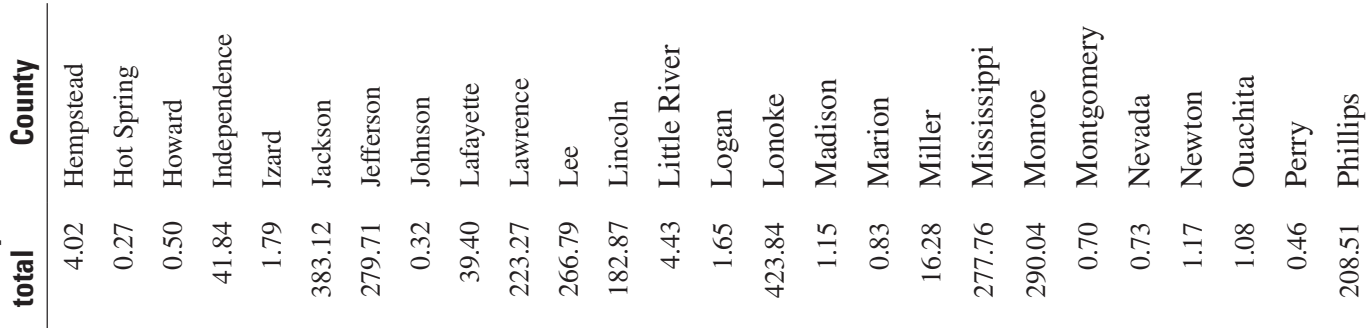

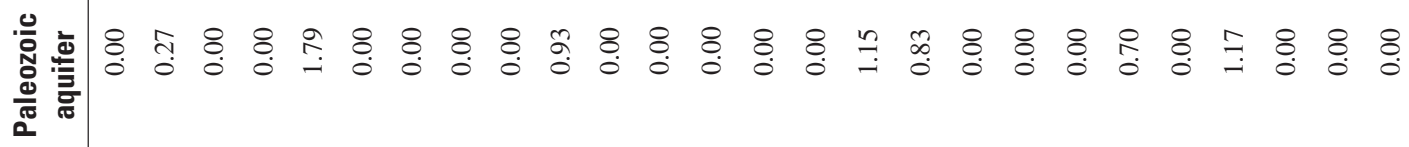

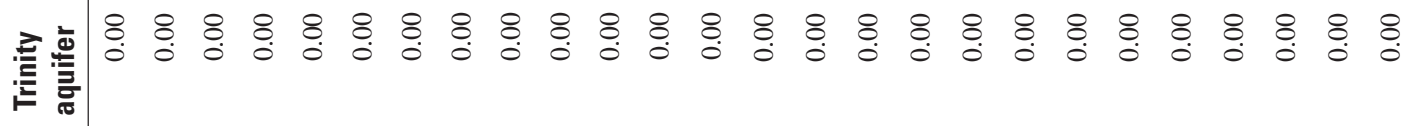

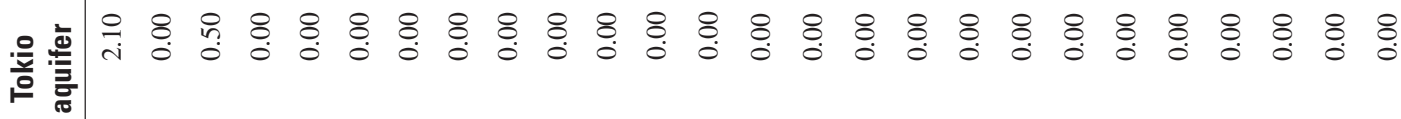

馬

竞

苨 उ

产高高

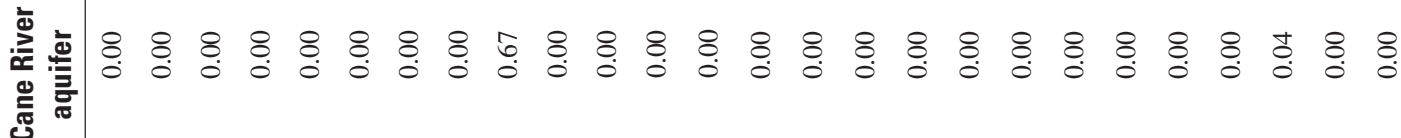

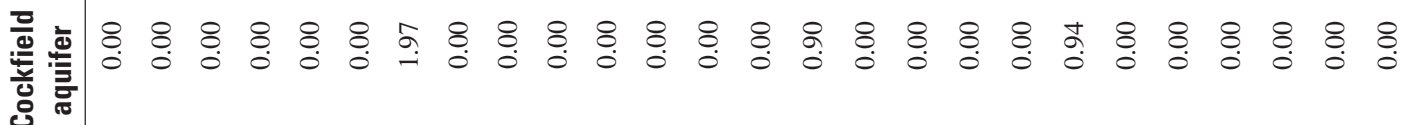

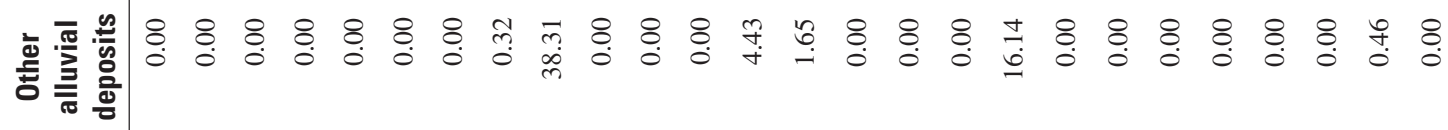

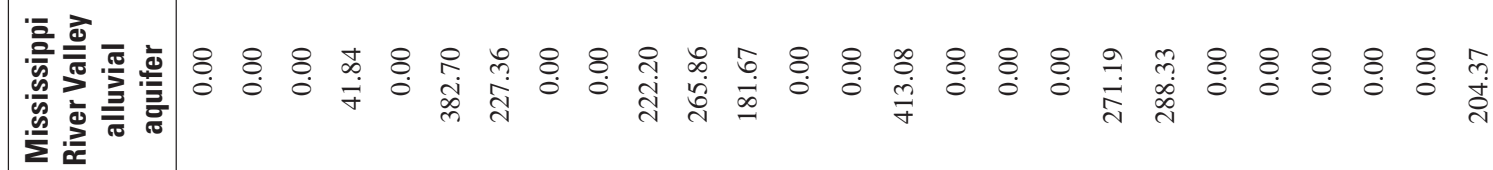

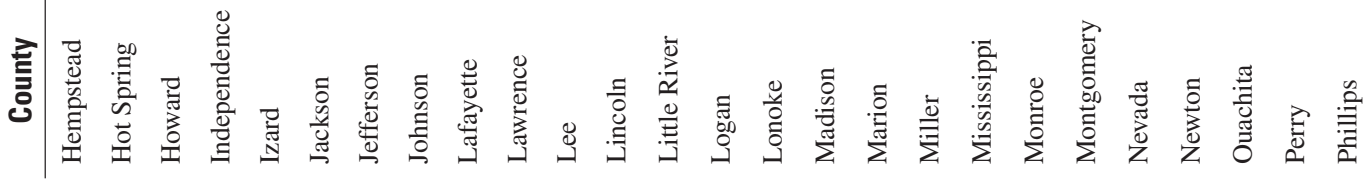




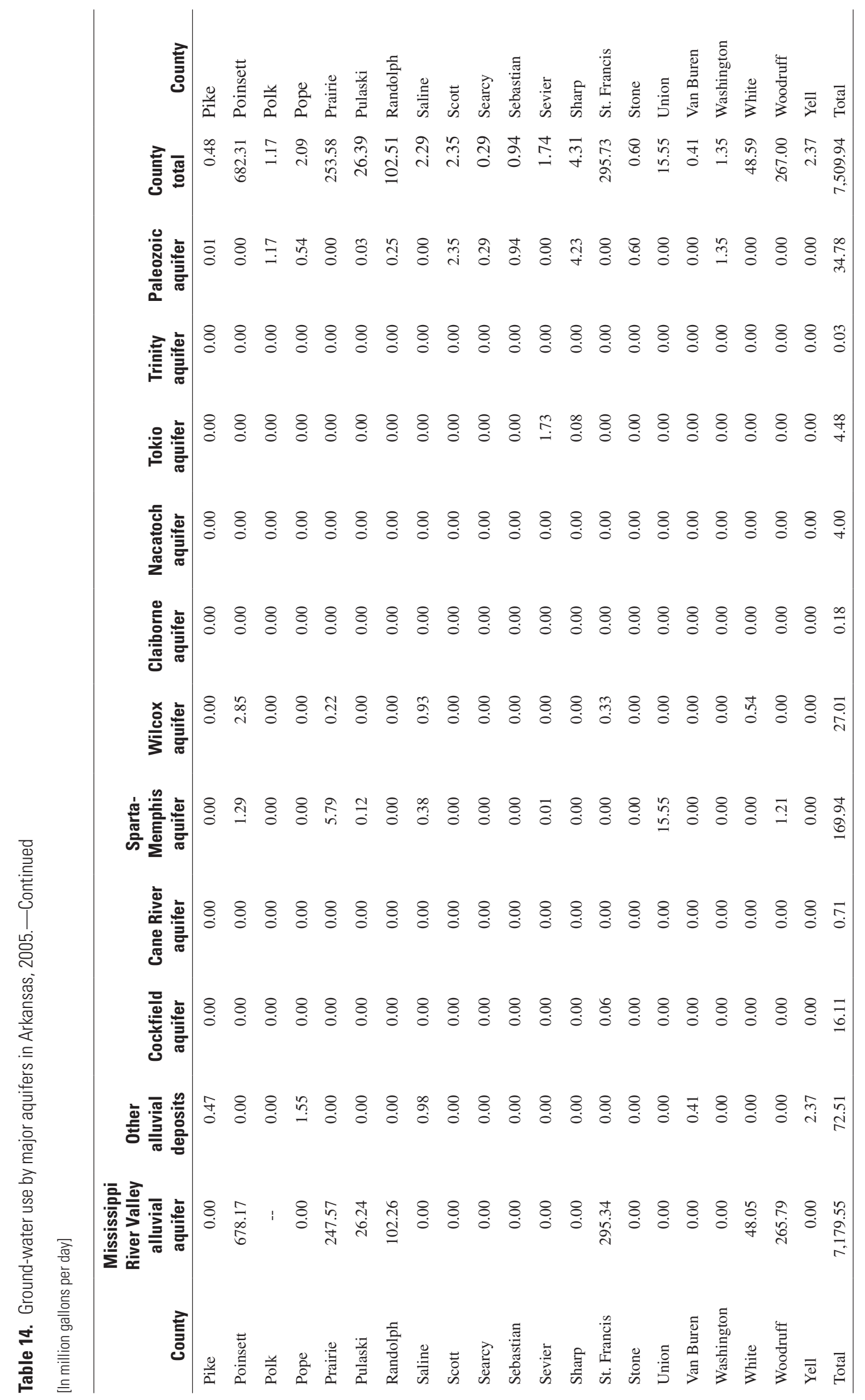




\section{Summary}

The water-use program in Arkansas is a cooperative effort between the Arkansas Natural Resources Commission and the U.S. Geological Survey to inventory water use. During 2005, the amount of water withdrawn from ground- and surface-water sources in Arkansas was estimated to be 11,455 $\mathrm{Mgal} / \mathrm{d}$. Of this amount, about 7,510 Mgal/d was from groundwater sources and about 3,946 Mgal/d was from surface-water sources.

Public supply systems served about 2.6 million people or about 93 percent of Arkansas' population in 2005. Benton County had the largest surface-water withdrawals (Beaver Lake) at $58 \mathrm{Mgal} / \mathrm{d}$. Jefferson County had the largest groundwater withdrawals (Sparta-Memphis aquifer) at $14 \mathrm{Mgal} / \mathrm{d}$. The statewide average for per capita residential use from public supply systems was about 157 gallons per day (gal/d). Carroll County had the highest per capita use (365 gal/d).

Total (self-supplied) industrial water use in Arkansas for 2005 was $178 \mathrm{Mgal} / \mathrm{d}$, of which about 37 percent $(66 \mathrm{Mgal} / \mathrm{d})$ was from ground-water and about 63 percent (113 Mgal/d) was from surface-water sources. The largest industrial use of ground water (Sparta-Memphis aquifer) occurred in Jefferson County (43 Mgal/d) and the largest industrial use of surface water occurred in Ashley County (53 Mgal/d).

Total aquaculture water use in Arkansas for 2005 was $256 \mathrm{Mgal} / \mathrm{d}$, of which about 96 percent (246 Mgal/d) was from ground water and about 4 percent $(11 \mathrm{Mgal} / \mathrm{d})$ was from surface-water sources. The largest use of aquacultural water was in Lonoke County at $60 \mathrm{Mgal} / \mathrm{d}$ of which 94 percent was from ground-water sources. The largest aquaculture use of surface water was in Lonoke County at $3.36 \mathrm{Mgal} / \mathrm{d}$. Other counties with notable aquaculture water use (greater than 20 $\mathrm{Mgal} / \mathrm{d}$ ) were Prairie and Ashley, in order of decreasing use.

Irrigation water use accounted for 92 percent $(6,942$ $\mathrm{Mgal} / \mathrm{d}$ ) of the ground water withdrawn in Arkansas and 72 percent $(8,265 \mathrm{Mgal} / \mathrm{d})$ of the total withdrawals. Rice totaled 1.73 million acres or about 35 percent of the total land irrigated in the State.

Irrigation water use totaled 8,265 Mgal/d, of which about 84 percent $(6,942 \mathrm{Mgal} / \mathrm{d})$ was from ground-water and about 16 percent (1,323 Mgal/d) was from surface-water sources. Water used for the growing of rice totaled 4,583 Mgal/d or about 40 percent of the total water used in Arkansas during 2005. The largest use of water for irrigation was Arkansas County with $836 \mathrm{Mgal} / \mathrm{d}$, of which about 56 percent (470 $\mathrm{Mgal} / \mathrm{d}$ ) was from ground-water and about 44 percent (366 $\mathrm{Mgal} / \mathrm{d}$ ) was from surface-water sources. Other counties with notable total irrigation water use (greater than $400 \mathrm{Mgal} / \mathrm{d}$ ) were Poinsett, Cross, Clay, and Lonoke, in order of decreasing use.

Duck (hunting) club water use in Arkansas for 2005 was $269 \mathrm{Mgal} / \mathrm{d}$, of which about 30 percent $(81 \mathrm{Mgal} / \mathrm{d})$ was from ground water and about 70 percent (188 Mgal/d) was from surface-water sources. The largest use of ground water for duck (hunting) clubs was in Arkansas County at $25 \mathrm{Mgal} / \mathrm{d}$. The largest duck (hunting) club use of surface water was in Arkansas County at $91 \mathrm{Mgal} / \mathrm{d}$. Other counties with substantial duck (hunting) club water use (greater than $10 \mathrm{Mgal} / \mathrm{d}$ ) were Miller, Desha, Prairie, Jackson, and Jefferson, in order of decreasing use.

Total thermoelectric power water use in Arkansas for 2005 was 1,997 Mgal/d of which over 99 percent (1,996 $\mathrm{Mgal} / \mathrm{d})$ was from surface water and less than 1 percent $(0.93$ $\mathrm{Mgal} / \mathrm{d}$ ) was from ground-water sources. Water used for thermoelectric power generation was about 17 percent of the total water used in Arkansas during 2005 and 51 percent of the total surface water used. About 58 percent $(1,152 \mathrm{Mgal} / \mathrm{d})$ of the water used in this category was used for cooling at a nuclear power plant in Pope County. About 98 percent (1,957 Mgal/d) of the water used in this category was used in Pope (Lake Dardanelle), Benton, Hot Spring (Lake Catherine and Ouachita River), Woodruff (White River), and Ouachita (Ouachita River) Counties, in order of decreasing use.

Total water use in Arkansas increased about 435 percent between 1965 and 2005. Total ground-water use increased 510 percent and total surface-water use increased 333 percent between 1965 and 2005.

About 98 percent of the ground water was withdrawn from the Mississippi River Valley alluvial and the SpartaMemphis aquifers. 


\section{Selected References}

Arkansas Department of Health, 2007, "Drinking Water Information for Arkansas," accessed February 2, 2007 at URL http://www.healthyarkansas.com/eng/pwslist0.htm.

Halberg, H.N., and Stephens, J.W., 1966, Use of water in Arkansas, 1965: Arkansas Geological Commission Water Resources Summary 5, 12 p.

Halberg, H.N., 1972, Use of water in Arkansas, 1970: Arkansas Geological Commission Water Resources Summary 7, $15 \mathrm{p}$.

Halberg, H.N., 1977, Use of water in Arkansas, 1975: Arkansas Geological Commission Water Resources Summary 9, $28 \mathrm{p}$.

Hall, A.P., and Holland, T.W., 1984, Water use in Arkansas, 1981: U.S. Geological Survey Water-Resources Investigations Report 84-4070, 1 sheet.

Holland, T.W., 1987, Use of water in Arkansas, 1985: Arkansas Geological Commission Water Resources Summary 16, $27 \mathrm{p}$.

Holland, T.W., 1993, Use of water in Arkansas, 1990: U.S. Geological Survey Open-File Report 93-48, 1 sheet.

Holland, T.W., 2004, Estimated Water Use in Arkansas, 2000; U.S. Geological Survey Scientific Investigations Report 2004-5230, 31 p.

Holland, T.W., 1999, Water Use in Arkansas, 1995: U.S. Geological Survey Open-File Report 99-188, 1 sheet.

Holland, T.W., and Hall, A.P., 1986, Water use in Arkansas, 1982: U.S. Geological Survey Water-Resources Investigations Report 85-4282, 1 sheet.

Holland, T.W., and Ludwig, A.H., 1981, Use of water in Arkansas, 1980: Arkansas Geological Commission Water Resources Summary 14, 30 p.

Hutson, S.A., and others, 2004, Estimated use of water in the United States in 2005: U.S. Geological Survey Circular $1268,46 \mathrm{p}$.

MacKichan, K.A., 1951, Estimated water use in the United States-1950: U.S. Geological Survey Circular 115, 13 p.

MacKichan, K.A., 1957, Estimated water use in the United States-1955: U.S. Geological Survey Circular 398, 18 p.

MacKichan, K.A., and Kammerer, J.C., 1961, Estimated water use in the United States, 1960: U.S. Geological Survey Circular 456, 26 p.

Murray, C.R., 1968, Estimated use of water in the United States, 1965: U.S. Geological Survey Circular 556, 53 p.
Murray, C.R., and Reeves, E.B., 1972, Estimated use of water in the United States, 1970: U.S. Geological Survey Circular $676,37 \mathrm{p}$.

Murray, C.R., and Reeves, E.B., 1977, Estimated use of water in the United States, 1975: U.S. Geological Survey Circular $765,37 \mathrm{p}$.

Petersen, J.C., Broom, M.E., and Bush, W.V., 1985, Geohydrologic units of the Gulf Coastal Plain in Arkansas: U.S. Geological Survey Water-Resources Investigations Report 85-4116, 20 p.

Solley, W.B., Chase, E.B., and Mann, W.B., 1983, Estimated use of water in the United States in 1980: U.S. Geological Survey Circular 1001, 56 p.

Solley, W.B., Merk, C.F., and Pierce, R.R., 1988, Estimated use of water in the United States in 1985: U.S. Geological Survey Circular 1004, 82 p.

Solley, W.B., Pierce, R.R., and Perlman, H.A., 1993, Estimated use of water in the United States in 1990: U.S. Geological Survey Circular 1081, 76 p.

Solley, W.B., Pierce, R.R., and Perlman, H.A., 1998, Estimated use of water in the United States in 1995: U.S. Geological Survey Circular 1200, 71 p.

Stephens, J.W., and Halberg, H.N., 1961, Use of water in Arkansas, 1960: Arkansas Geological Commission Water Resources Special Report Series 294, 56 p.

U.S. Bureau of Commerce, U.S. Census Bureau, 2005: Census 2005 summary file (SF1) 100-percent data, accessed December 1, 2006, at http://factfinder.census.gov

U.S. Department of Agriculture, 2005, Crop Reporting Service web site, accessed July 17, 2006 at http://www.usda.gov/nass/pubs/agr05/acro05.htm. 


\section{Glossary}

Water-use terminology continues to evolve as the field grows and expands. The following is a list of definitions for terms, phrases, and various data-collection components commonly used in the process of water-use data collection and compilation.

Acre-foot of water (acre-ft) - the volume of water required to cover 1 acre of land $(43,560 \mathrm{ft} 2)$ to a depth of $1 \mathrm{ft}$.

Acres irrigated - the total number of acres of the crop that was irrigated during the year.

Aquaculture - Farming of organisms that live in water, such as fish, shellfish, and algae.

Aquifer-A geologic formation, group of formations, or part of a formation that contains sufficient saturated permeable material to yield significant quantities of water to wells and springs.

Commercial water use-Water for motels, hotels, restaurants, office buildings, schools, and other commercial facilities and institutions, both civilian and military. The water may be obtained from a public-supply facility or may be self-supplied. See also public-supply and self-supplied water.

Cooling water-Water used for cooling purposes, such as cooling of condensers and nuclear reactors.

Domestic population served-The total number of people served by the public supplier during the calendar year.

Domestic water use-Water used for household purposes, such as drinking, food preparation, bathing, washing clothes and dishes, flushing toilets, and watering lawns and gardens, also called residential water use. The water may be obtained from a public-supply facility or may be self-supplied. See also public supply and self-supplied water.

Freshwater-Water that contains less that $1,000 \mathrm{mg} / \mathrm{L}$ (milligrams per liter) of dissolved solids and is desirable for drinking and many industrial uses.

Ground water-Generally all subsurface water and springs as distinct from surface water; specifically, that part of the subsurface water in the saturated zone (a zone in which all voids are filled with water) where the water is under pressure greater than atmospheric.
Industrial water use - Water used for industrial purposes, such as fabrication, processing, washing, and cooling, and includes such industries as steel, chemical and allied products, paper and allied products, mining, and petroleum refining. The water may be obtained from a public-supply facility or may be selfsupplied. See also public-supply and self-supplied water.

Instream use-Water use taking place within the stream channel for such purposes as hydroelectric power generation, navigation, water-quality improvement, fish propagation, and recreation. Sometimes called nonwithdrawal use or in-channel use.

Irrigation-Refers to the process of supplementing rainfall with water that is needed to produce a crop.

Irrigation water use-Artificial application of water on lands to assist in the growing of crops and pastures or to maintain vegetative growth in recreational lands, such as parks and golf courses.

Livestock water use-Water for stock watering, feed lots, dairy operations, fish farming, and other farm needs. Livestock as used here includes cattle, sheep, goats, hogs, and poultry. Also included are such animal specialties as horses, rabbits, bees, pets, and fur-bearing animals in captivity.

Mining water use-Water use for the extraction of minerals occurring naturally, including solids, such as coal, clay, and ores; liquids, such as crude petroleum; and gases, such as natural gas. Also includes uses associated with sand, gravel, and quarrying for rock aggregates, well operations (dewatering), milling (crushing, screening, washing, floatation, and other), and other preparations customarily done at the mine site or as part of a mining activity.

Per capita use - The average amount of water used per person during a standard time period, generally per day.

Public supply - Water withdrawn by public and private water suppliers and delivered to groups of users. Public suppliers provide water for a variety of uses, such as domestic, commercial, thermoelectric power, industrial, and public water use. See also commercial water use, domestic water use, industrial water use, and public water use.

Public water use-Water supplied from a public water supply and used for such purposes as firefighting, street washing, and municipal parks and swimming pools. See also public supply. 
Self-supplied water-Water withdrawn from a ground- or surface-water source by a user rather than being obtained from a public-supply facility.

Sewage-Wastewater carried off by sewers and drains. Surface water-An open body of water, such as a stream, lake, or river.

Thermoelectric power generation-Electrical power generated using fossil-fuel (coal, oil, or natural gas), geothermal, or nuclear energy.

Thermoelectric power water use-Water used in the process of the generation of thermoelectric power. The water may be obtained from a public-supply facility or may be self-supplied. See also public-supply and self-supplied water.

Water consumed or consumptive use - Refers to that part of water withdrawn that is no longer available because it has evaporated, been incorporated into products and crops, consumed by man or livestock, or otherwise removed from the water environment.

Water use-Describes how and where the water was used and in what amounts.

Withdrawal-The amount of water withdrawn from a source (ground water or surface water). This is equivalent to "intake," "water diversion," or "pumpage," terms commonly used by industry and for irrigation and public supply, respectively.

Publishing support provided by:

Rolla Publishing Service Center

For more information concerning the research described

in the report:

U.S. Geological Survey

Arkansas Water Science Center

401 Hardin Road

Little Rock, AR 72211-3528

(501) 228-3600

http://ar.water.usgs.gov 
